\title{
CONTRIBUCIÓN A LA CLASIFICACIÓN FITOSOCIOLÓGICA DE LOS PASTIZALES DE LA PROVINCIA DE CÁDIZ (ESPAÑA)
}

\author{
Antonio GALÁN DE MERA, Ulrich DEIL, Horst HAUG y José A. VICENTE ORELLANA
}

\begin{abstract}
RESUMEN. Contribución a la clasificación fitosociológica de los pastizales de la provincia de Cádiz (España). En el presente trabajo se estudian las comunidades de pastos del centro y suroeste de la provincia de Cádiz en base a 521 inventarios fitosociológicos. Todos ellos han sido sometidos a dos análisis multivariantes, uno de ordenación (PCA) y otro de clasificación. Con ambos métodos se han obtenido 50 grupos de vegetación correspondientes a asociaciones pertenecientes a diferentes clases fitosociológicas: Corynephoretea canescentis, Poetea bulbosae, Molinio-Arrhenatheretea, Polygono arenastri-Poetea annuae, Ruderali-Secalietea cerealis, Juncetea bufonii, Littorelletea, Phragmitio-Magnocaricetea y Frankenietea pulverulentae. Como resultado del tratamiento estadístico se describe un nuevo ordenPhalaridetalia coerulescentis, una alianza -Gaudinio fragilis-Hordeion bulbosi, tres asociaciones Deschampsio strictae-Agrostietum curtisii, Hedysaro coronarii-Phalaridetum coerulescentis y Poo sylvicolae-Festucetum atlantigenae y dos subasociaciones -Gaudinio fragilis-Agrostietum castellanae hypochaeridetosum platylepidis y Poo sylvicolae-Festucetum atlantigenae scirpetosum maritimi.
\end{abstract}

Palabras clave. Pastizales, fitosociología, Cádiz, España.

ABSTRACT. Contribution to the phytosociological classification of the grasslands of the province of Cadiz (Spain). In this paper the grasslands communities of the centre and the southwestern of the province of Cadiz are studied on the base of 521 phytosociological relevés. After a multivariate analysis, ordination (PCA) and classification, 50 vegetation types have been defined, corresponding to different phytosociological classes: Corynephoretea canescentis, Poetea bulbosae, Molinio-Arrhenatheretea, Polygono arenastriPoetea annuae, Ruderali-Secalietea cerealis, Juncetea bufonii, Littorelletea, Phragmitio-Magnocaricetea y Frankenietea pulverulentae. As a result of the statistics treatment, a new order-Phalaridetalia coerulescentis, an alliance-Gaudinio fragilis-Hordeion bulbosi, three associations -Deschampsio strictaeAgrostietum curtisii, Hedysaro coronarii-Phalaridetum coerulescentis and Poo sylvicolae-Festucetum atlantigenae and two subassociations -Gaudinio fragilis-Agrostietum castellanae hypochaeridetosum platylepidis and Poo sylvicolae-Festucetum atlantigenae scirpetosum maritimi are described.

Key words. Grasslands, phytosociology, Cadiz, Spain.

\section{INTRODUCCIÓN Y OBJETIVOS}

Los pastizales del SO de la Península Ibérica han sido escasamente estudiados desde el punto de vista fitosociológico. Aparte de los datos que aparecen en la obra general de Rivas Goday \& Rivas-Martínez (1963), se pueden destacar algunos trabajos sobre comunidades

Estudio realizado en el marco de los proyectos DE 402 del DFG (Alemania) y 12/95 de la Universidad San Pablo-CEU (España) 
anuales (Galán de Mera et al., 1995) y ciertas aportaciones sobre las fitocenosis vivaces (Rivas-Martínez et al., 1980; Bartolomé et al., 1989; Nezadal et al., 1994). De otro lado, Fernández Alés et al. (1991) realizan un análisis multivariante en pastizales de la provincia de Cádiz distinguiendo dos grandes unidades aunque sin precisar la importancia fitocenológica de los taxones. Sin embargo, precisamente las técnicas multivariantes han demostrado ser una herramienta muy útil en fitosociología (Holzner et al., 1978; Moore \& Sullivan, 1978; Orlóci, 1978; Van der Maarel, 1979) especialmente para objetivizar las tendencias de las plantas con las variaciones de determinados parámetros ecológicos.

El objetivo de este trabajo es emplear las técnicas multivariantes para clasificar a los pastizales en unidades fitosociológicas o sintaxones.

\section{ÁREA DE ESTUDIO}

Los territorios donde se ha realizado el estudio se encuentran en el centro y suroeste de la provincia de Cádiz, en el sur de España (fig. 1). Según las últimas aproximaciones fitogeográficas para el sur de la Península Ibérica (Rivas-Martínez et al., 1991; Galán de Mera \& Vicente Orellana, 1996; Pérez Latorre et al., 1996) el área participa de la provincia Tingitano-Onubo-Algarviense con los sectores Aljíbico (subsectores Aljíbico, Sidonense y Algecireño) y Gaditano-Onubense. Según la clasificación bioclimática y sucesional de la vegetación de Rivas-Martínez (1987), son dos las series climatófilas que encabezan la vegetación del sector Aljíbico: los alcornocales y quejigares africanos (Teucrio baeticiQuerceto suberis sigmetum, Rusco hypophylliQuerceto canariensis sigmetum) que, bajo un bioclima termo-mesomediterráneo húmedohiperhúmedo pueblan las areniscas miocenas del Aljibe (Chauve, 1968). La serie edafófila

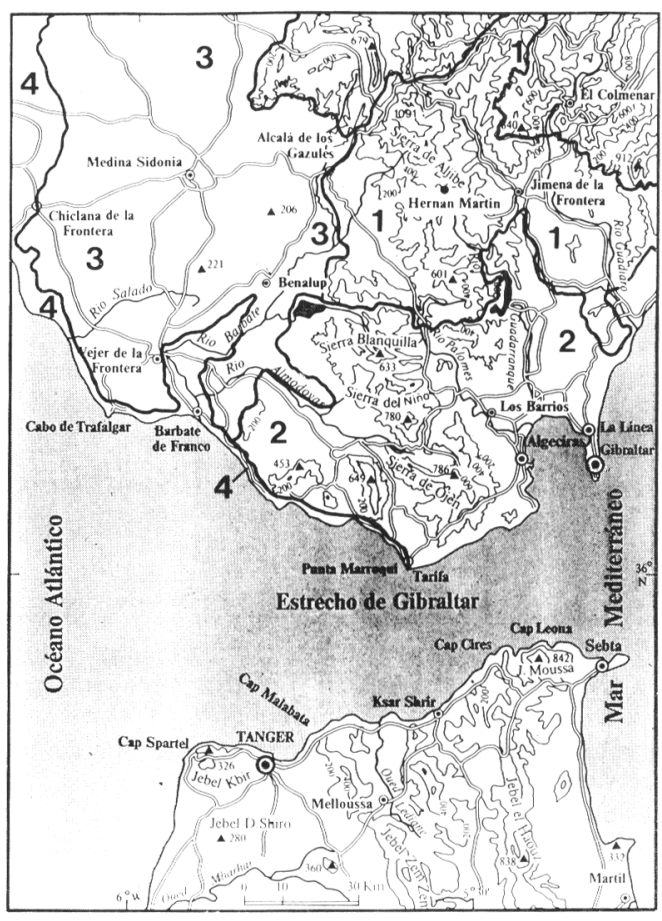

Figura 1. Mapa del territorio estudiado y su sectorización fitogeográfica. Sector Aljíbico : 1 subsector Aljíbico, 2- subsector Algecireño, 3subsector Sidonense. Sector Gaditano-Onubense : 4- subsector Gaditano-Litoral. Map of the studied territory and its phytogeographical sectoring. Aljibico sector: 1-Aljibico subsector, 2-Algecireño subsector, 3-Sidonense subsector. GaditanoOnubense sector : 4-Gaditano-Litoral subsector.

verticícola termomediterránea subhúmedohúmeda del acebuche (Tamo communis-Oleeto sylvestris sigmetum) también está ampliamente representada en este sector, especialmente en el subsector Sidonense. Las arenas costeras con alcornocales psamófilos- serie termomediterránea seco-subhúmedo-húmeda del alcornoque (Myrto communis-Querceto suberis halimietoso sigmetum) y sus etapas de sustitución con los matorrales de Stauracanthus genistoides, Thymus albicans y Ulex australis, ponen de manifiesto los límites del sector 
Gaditano-Onubense. La provincia TingitanoOnubo-Algarviense, además, comprende a otras comarcas que se extienden hasta la desembocadura del río Aveiro (Portugal) por el norte, y hasta el Bajo Loukkos (Marruecos), en el norte de Africa.

\section{MATERIAL Y MÉTODOS}

Se efectuaron. 521 inventarios fitosociológicos de comunidades de pastos desarrolladas sobre diferentes suelos (Carballas et al., 1981; Mudarra Gómez et al., 1989) y con distintos usos. Las diferentes comunidades se dividieron en vivaces y anfibias, nitrófilas y juncales. Para cada taxon se realizó una estima de su abundancia-dominancia sobre una escala ponderada $(r,+, 1,2,3,4,5)$ propuesta por Braun-Blanquet (Braun-Blanquet, 1979). Con el fin de sistematizar la información para el tratamiento automático de datos, éstos se transformaron a una escala ordinal siguiendo a Van der Maarel (1979): r-1, +-2, 1-3, 2-4, 3-5, 4-6, 5-7. Por otro lado, en cada inventario se anotó la superficie inventariada y la altitud. En la matriz resultante no se tuvieron en cuenta las plantas compañeras cuya presencia era dos o menor de dos para evitar «ruido» durante el tratamiento de los datos (Pielou, 1969). El carácter de «compañeras» o de características de los diferentes sintaxones, ha sidn determinado según anteriores trabajos donde se incluyen pastos europeos y norteafricanos (Gounot, 1958; Tüxen \& Oberdorfer, 1958; Rivas Goday, 1964; González et al., 1973; Rivas-Martínez et al., 1980; Rivas-Martínez et al., 1984; Rivas-Martínez et al., 1986; Santos et al., 1989; Oberdorfer, 1990; Rivas-Martínez et al., 1991b). Dicha matriz se procesó con el paquete estadístico ANACOM V. 3.0 (De la Cruz, 1991) y tanto para la ordenación como para la clasificación se empleó el índice de Bray \& Curtis (1957).

\section{RESULTADOS Y DISCUSIÓN}

\section{Análisis estadístico}

Como resultado del análisis estadístico se han obtenido grupos de comunidades de plantas que se corresponden con las diferentes asociaciones que se tratan. En las figuras 2, 3 y 4 se muestran los dendrogramas resultantes de la clasificación, que indican el grado de similitud que existe entre las diferentes comunidades vegetales. En las figuras 5, 6 y 7 se ofrece el resultado del análisis de componentes principales (PCA) sobre los ejes I y II donde, como se puede observar, aparecen nubes de puntos que separan a las diferentes asociaciones según su composición florística.

\subsection{Comunidades vivaces y anfibias (Juncetea bufonii)}

En el dendrograma de la figura 2, el primer grupo que se separa es A-Deschampsio strictae-Agrostietum curtisii. Es un pastizal bien distinto al resto de los considerados al estar caracterizado por gramíneas xerófilas de carácter atlántico. B y $\mathrm{M}$, son los prados con Plantago serraria (Trifolio subterraneiPlantaginetum serrariae). Ambos grupos se encuentran en el mismo nivel de disociación aunque distantes. En el dendrograma M está junto a L (comunidades con Phalaris coerulescens), y en el PCA (fig. 5), M y L coinciden con los inventarios imbricados a la derecha del eje I. Esto es explicable porque los prados de Poetea bulbosae se enriquecen de plantas transgresivas debido al empleo rotativo de los pastos en el territorio. B, C y D también se encuentran muy relacionados en ambos análisis. C son los vallicares de Agrostis castellana, que dan paso a las comunidades de Poetea bulbosae en los biótopos donde afloran arcillas y hay un constante pisoteo del ganado vacuno. D son los vallicares efímeros hidromorfos con Agrostis pourretii (=Agrostis salmantica). Los grupos E a L significan los pastos más extendidos en la provincia de Cádiz 


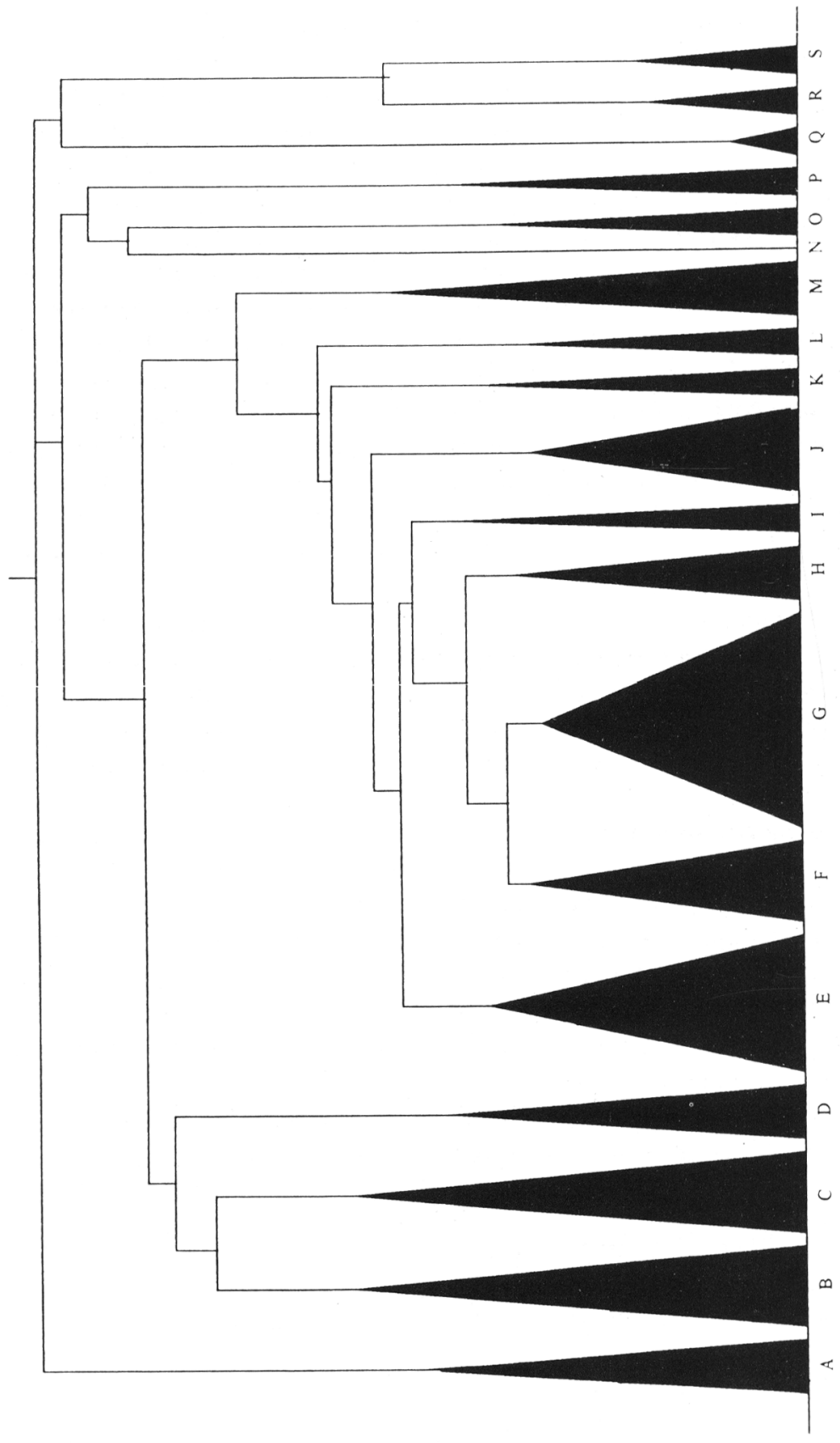

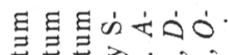

\section{.}

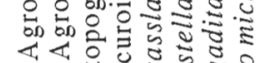

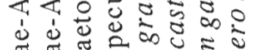

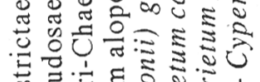

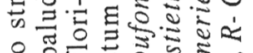
으를 क.

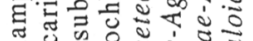
यू.

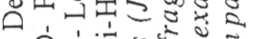

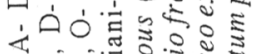
4. :

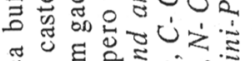

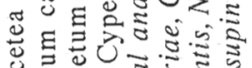

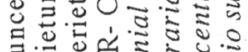

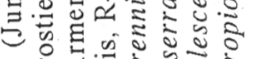

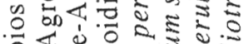

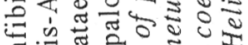

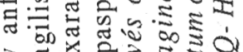

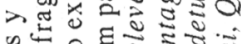

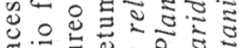

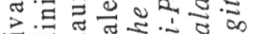

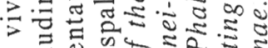

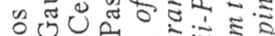

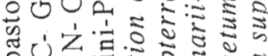

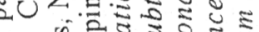

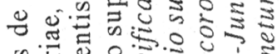
क

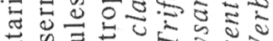

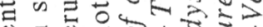
园:0 寻苟出

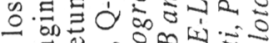

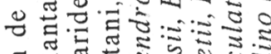

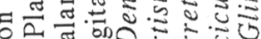

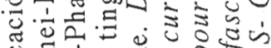

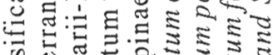

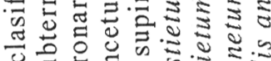

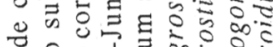
으의

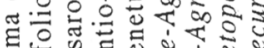
त्. की

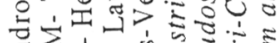

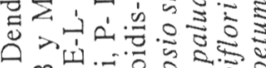
i. $m=0$

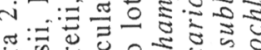

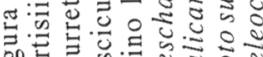

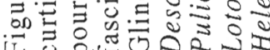


que están presididos por Phalaris coerulescens. Dentro de ellos, hay dos grupos que se disocian un poco más a la derecha del eje del dendrograma, K y L. K es una variante con Leontodon longirostris y Cynara humilis; $\mathrm{L}$ es otra variante enriquecida con Leontodon maroccanus.

El prado con Armeria gaditana es el grupo $\mathrm{N}$ (Centaureo exaratae-Armerietum gaditanae). Tanto $\mathrm{C}$ como $\mathrm{N}$, pertenecen a la alianza Agrostion castellanae. Aunque en el PCA se encuentran próximos a la izquierda del eje I, en el dendrograma están distantes debido a que las asociaciones de contacto son muy diferentes. Mientras que $\mathrm{C}$ se muestra en contacto con el Pulicario-Agrostietum pourretii (D), N con Loto-Chaetopogonetum fasciculati (O).

Los grupos P, Q, R y S son el resto de las asociaciones de Juncetea bufonii (P, LaurentioJuncetum tingitani; Q, Heliotropio supiniPaspaletum paspaloidis; R, Cypero michelianiHeleochloetum alopecuroidis; S, Glino lotoidis-Verbenetum supinae). Q, R y S están separados en una fracción del dendrograma diferente a P. Esto es debido a la diferente fenología de las asociaciones. Mientras que Laurentio-Juncetum tingitani es primaveral, Q, R y S son tardo-estivales y otoñales.

\subsection{Comunidades nitrófilas}

Tanto en el dendrograma (fig. 3) como en el PCA (fig. 6) aparecen los grandes grupos de comunidades bien diferenciados. Sin considerar a las asociaciones menos representadas en el paisaje, en ambas versiones estadísticas hay dos grandes complejos: B, C, D y Easociaciones estivo-autumnales y F-Qasociaciones vernales. En éstas últimas hay que destacar a las comunidades subnitrófilas de Echio-Galactition (Trifolio pallidiVulpietum geniculatae) bastante extendidas por todos los suelos areno-limosos húmedos del territorio (Galán de Mera, 1995)[G a J]. En los extremos del dendrograma tenemos a las comunidades más peculiares: A-Plantagini coronopi-Hordeetum marini (sobre las facies salinas del Keuper), R- Kickxio lanigeraeTanacetetum annui (vegetación autumnal de barbechos inundables)[Galán de Mera, 1996], S- Comunidad de Setaria verticillata y Echinochloa hispidula (huertas y suelos bien irrigados).

\subsection{Los juncales}

En el dendrograma (fig. 4) hay dos grandes ramas: A-I y J, K, L. La primera son los juncales y herbazales de los medios más húmedos; J, K y $\mathrm{L}$ son las comunidades asentadas sobre los suelos que soportan una mayor sequía. A y B son dos comunidades que llevan en su composición plantas turfófilas: Hydrocotyle vulgaris, Hypericum undulatum, Juncus striatus, Lotus uliginosus, Prunella vulgaris y Silene laeta. C y D se desarrollan sobre suelos arenosos compactados y pastoreados. D- llega a ser una versión más húmeda de C (Trifolio resupinati-Caricetum chaetophyllae) donde Eleocharis palustris aumenta su presencia. E y F es la asociación Glycerio declinataeEleocharitetum palustris que son humedales de aguas someras, temporales y pobres en bases. $\mathrm{G}$ aparece algo separado del resto de las asociaciones al llevar una planta dominante bien diferente-que destaca en las depresiones arcillosas salobres, Festuca arundinacea subsp. atlantigena. $\mathrm{H}$ es la comunidad turfófila de Eleocharis multicaulis; en el dendrograma se encuentrabien distanciada de A y B que también se asientan sobre suelos poco oxigenados. Esto es debido a que $\mathrm{H}$ muestra en el territorio tendencia hacia los medios más nitrificados, 10 que se demuestra por el contacto con las berreras de Apium nodiflorum [I- Glycerio declinatae-Apietum nodiflori Molina (1996)]. Sin embargo, en el PCA (fig. 7), A, B y $\mathrm{H}$ aparecen agrupados juntos en la parte superior izquierda del gráfico. J, K y L es la vegetación que ocupa la banda más externa de los humedales según la siguiente secuencia hacia 


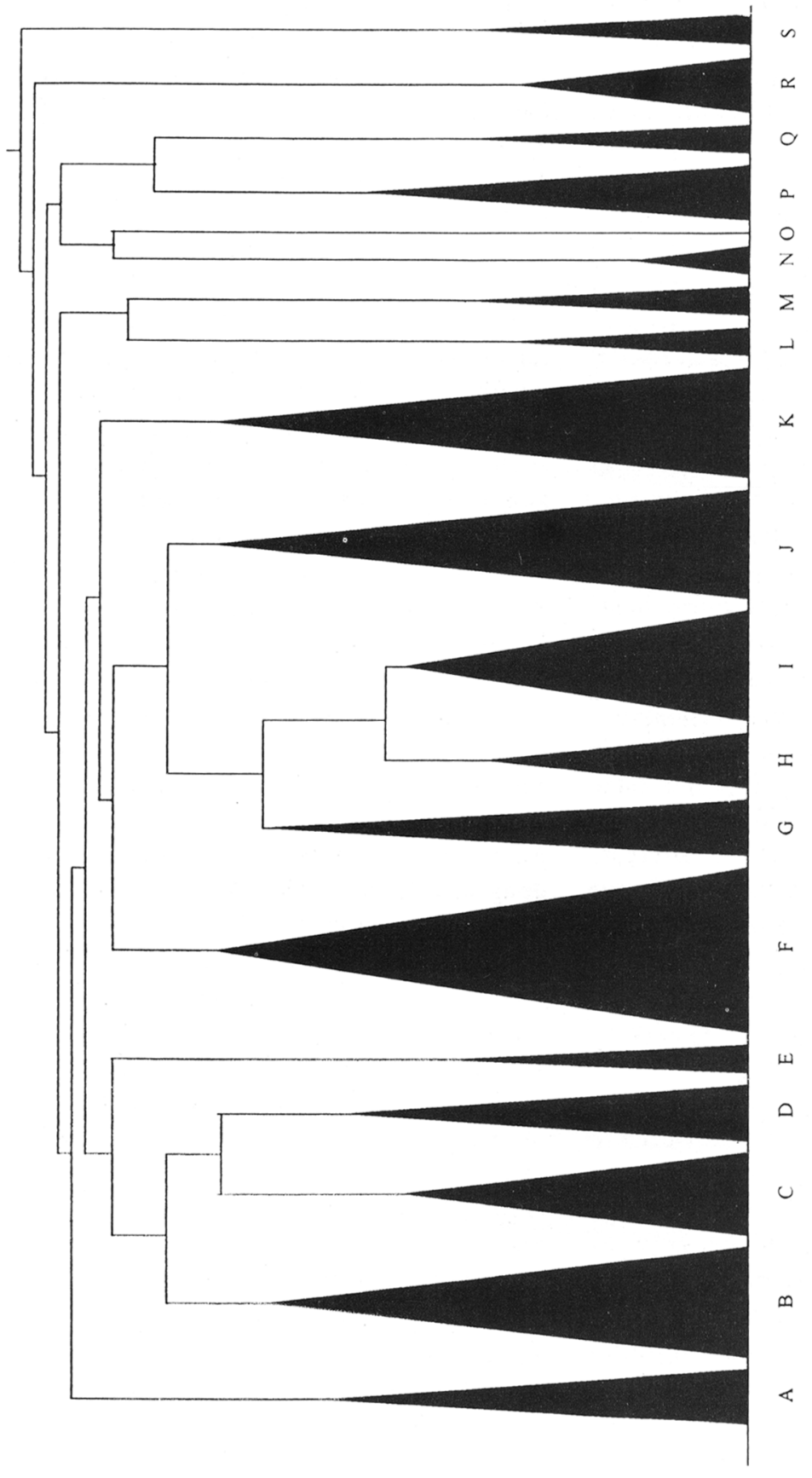

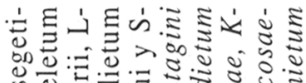

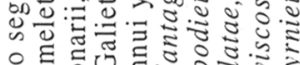

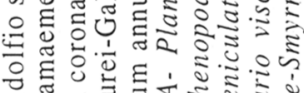

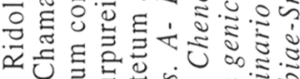

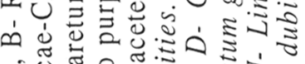

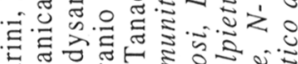

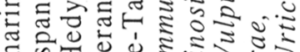
ह.气 ह 至을.

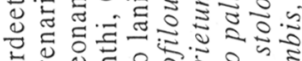

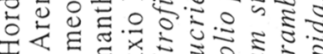

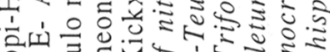

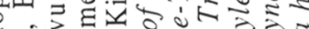

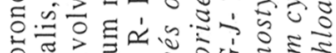

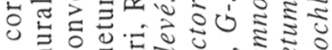

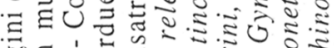

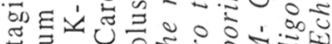

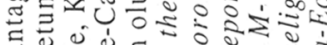

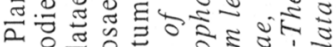
1.

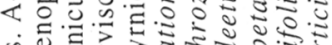

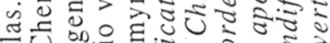

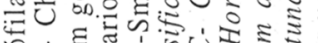

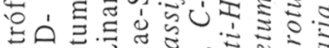
草.

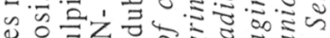

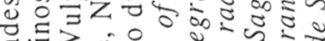
ขु.

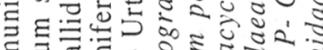

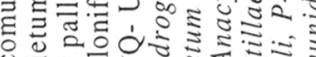
范의의

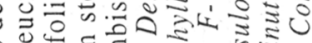

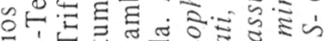

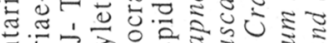

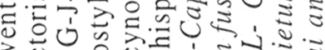

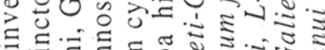

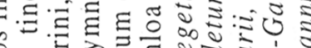

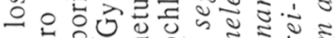

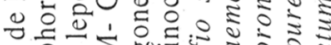

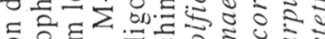

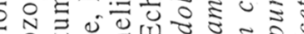

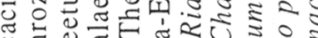

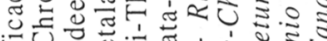
虫

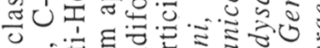

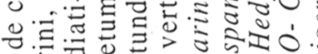

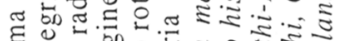

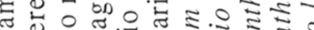

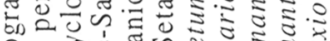

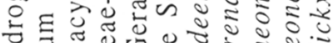

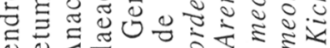

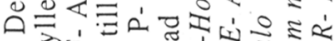

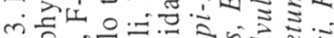

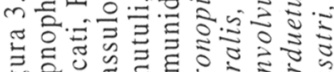

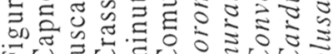


los suelos más secos: J- Galio palustriJuncetum maritimi, K-Holoschoeno-Juncetum acuti, L-Astero squamati-Panicetum repentis. Esta última es una comunidad mesonitrófila estival que en muchas ocasiones se instala en los suelos más secos de los humedales.

\section{Discusión sintaxonómica}

Como resultado del análisis estadístico y de la comparación de las tablas fitosociológicas con las observaciones de otros autores, describimos los sintaxones que se enumeran a continuación:

\subsection{Deschampsio strictae-Agrostietum curtisii ass. nova}

[Typus: Tabla 1, invent. 5]

Graminales xerófilos mesomediterráneos desarrollados sobre rankers y en áreas con ombroclima húmedo-hiperhúmedo de la sierra del Aljibe. Son plantas características de la asociación: Avenula sulcata subsp. albinervis y Festuca baetica. A Satureja salzmannii podemos considerarla como una buena diferencial. Las plantas que encontramos en su composición florística están con frecuencia en la bibliografía en el seno de alcornocales o de formaciones con Quercus lusitanica (RivasMartínez, 1979; Barbero et al., 1981; RivasMartínez et al., 1990) aunque tienen su óptimo en áreas puntuales culminícolas formando céspedes poco extensos.

De las tres alianzas del orden Jasiono sessiliflorae-Koelerietalia crassipedis existentes en la Península Ibérica (HieracioPlantaginion radicatae Rivas-Martínez \& Cantó 1986- meso-supramediterránea, carpetano-ibérico-leonesa; Agrostio castellanae-Stipion giganteae Rivas Goday (1957) 1964- meso-supramediterránea, carpetano-ibérico-leonesa y lusoextremadurense; Festucion elegantis RivasMartínez \& Sánchez Mata 1986- mesosupramediterránea, iberomarroquí-atlántica) Festucion elegantis reune a los pastizales meso- supramediterráneos de areal iberomarroquíatlántico, por lo que adscribimos a ella esta nueva asociación. Son características territoriales de la alianza: Agrostis curtisii, Avenula sulcata subsp. albinervis, Deschampsia stricta y Festuca baetica.

\subsection{Hedysaro coronarii-Phalaridetum coerulescentis ass. nova}

[Typus: Tabla 1, invent. 14]

Prados vivaces caracterizados por abundantes gramíneas bulbosas (Hordeum bulbosum, Phalaris coerulescens) y leguminosas (Hedysarum coronarium, Trifolium baeticum, T. isthmocarpum, T. squamosum) que les confieren un buen valor pastoral (Gounot, 1958; Thiault, 1957 y 1961). La estructura de la comunidad viene dada por Hordeum bulbosum y Phalaris coerulescens que llegan a alcanzar hasta $1 \mathrm{~m}$ de altura, haciéndose rastreras en el suelo el resto de las plantas forrageras, entre las que resalta Hedysarum coronarium por su abundancia e inflorescencias vistosas y purpúreas.

Esta biocenosis se asienta sobre suelos profundos tirsificados, tanto de naturaleza ácida como básica, quedando encuadrada en el bioclima termomediterráneo subhúmedohiperhúmedo de los sectores Aljíbico, Tingitano y zonas occidentales de la provincia Bética. Además, su óptimo está en territorios con una alta humedad oceánica, que se hace patente a lo largo de todo el año, y hace que los períodos asimiladores de las plantas sean largos, con un crecimiento vegetal que compensa cualquier mutilación por pastoreo (Montserrat, 1981).

La dinámica y uso de estos pastizales han sido estudiados fundamentalmente por Gounot (1958) y González de Tanago et al. (1980). En la provincia de Cádiz son usados con pastoreo contínuo hasta principios del verano, o bien son sometidos a sistemas de siega con fines de henificación. Este último aspecto los aproxima a las praderas de siega eurosiberianas (Rivas- 


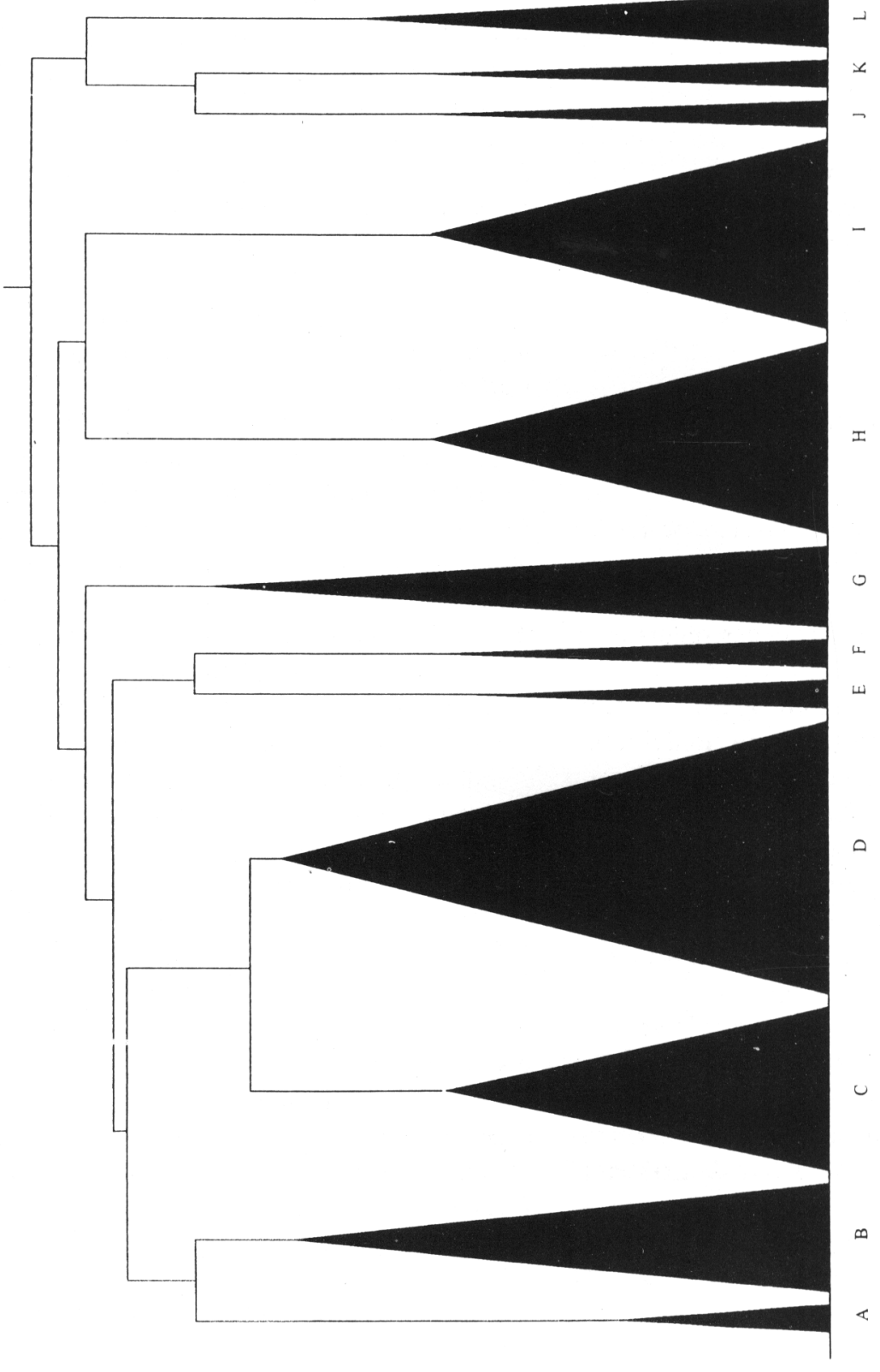


Martínez et al., 1984). Sin embargo, estos graminales no dejan de ser muy particulares; no hay un proceso de encespedado como sucede en la Poetea debido a las dilataciones del suelo por sequía-hidratación (Montserrat, 1981). Su origen es el ahuecado y uso anterior del suelo con cultivos que al ser abandonados permiten la instalación de un herbazal nitrófilo con formas anuales pioneras de Hedysarum coronarium; estas tierras posías abandonadas con un herbazal nitrófilo que comienza a ser pastoreado, se transforma en un período de 2 a 4 años en la pradera de Hordeum y Phalaris cuyas características se mantienen únicamente con la siega a finales del verano.

Podemos distinguir dos variantes de este pastizal debidas a su uso: variante con Leontodon longirostris y Cynara humilis, propia de áreas sin un cuidado regular, y variante con Leontodon maroccanus, de áreas que han sido cuidadas con siegas regulares y sitios más húmedos.

Las características ecológicas y florísticas que presenta este pastizal lo separan del resto de las comunidades vivaces eurosiberianas, por lo que proponemos la creación de una alianza y un orden nuevos dentro de la clase Molinio-Arrhenatheretea.

\subsubsection{Gaudinio fragilis-Hordeion bulbosi all. nova}

[Typus: Hedysaro coronarii-Phalaridetum coerulescentis ass. nova]

La presencia de un alto número de plantas de areal mediterráneo-occidental e incluso bético, aljíbico y tingitano, permiten diferenciar esta nueva alianza, que engloba a las comunidades que se desarrollan sobre suelos profundos vérticos y con fuertes encharcamientos estacionales. Los taxones característicos son: Hedysarum coronarium, Hypochaeris radicata subsp. platylepis, Iris planifolia, Leontodon maroccanus, Scorzonera baetica, Scrophularia sambucifolia subsp. mellifera, S. sambucifolia subsp. sambucifolia,
Stachys germanica subsp. cordigera y Trifolium baeticum. Es una alianza mediterránea occidental y central que, hasta el momento agrupa las asociaciones siguientes: Hedysaro-Phalaridetum coerulescentis ass. nova, Phalarido coerulescentis-Agropyretum pungentis Loppi \& De Dominicis 1990 y Phalarido coerulescentis-Dactyletum glomeratae Scoppola \& Pelosi 1995 (centro de Italia).

\subsubsection{Phalaridetalia coerulescentis ordo novo [Typus: Gaudinio fragilis-Hordeion bulbosi all. nova]}

Las praderas de la clase MolinioArrhenatheretea son de óptimo eurosiberiano (Oberdorfer, 1990; Dierschke, 1995) aunque presentan irradiaciones hacia las áreas más atlánticas de la Región Mediterránea a través del orden Arrhenatheretalia Pawlowski 1928 (Koch, 1926; Tüxen \& Oberdorfer, 1958; RivasMartínez et al., 1984). Numerosas especies de la clase se encuentran también en las comunidades de Hedysarum coronarium y Phalaris coerulescens: Carex distans, Carex divisa, Crepis capillaris, Hordeum geniculatum, Lythrum junceum, Mentha pulegium, Parentucellia viscosa, Plantago lanceolata, Poa trivialis, Ranunculus trilobus, Rumex conglomeratus y Rumex crispus. Sin embargo, estos prados meridionales, de áreas oceánicas, tienen un papel ecológico semejante a la Arrhenatheretalia eurosiberiana pero tiene un cortejo florístico mediterráneo y paleomediterráneo (Islas Macaronésicas): Hordeum bulbosum, Narcissus papyraceus, Phalaris aquatica, Phalaris coerulescens, Phalaris truncata, Scilla peruviana.

\subsection{Poo sylvicolae-Festucetum atlantigenae ass. nova}

[Typus: Tabla 1, invent. 44]

Asociación presidida por Festuca arundinacea subsp. atlantigena que puebla depresiones encharcadas hasta principios del 


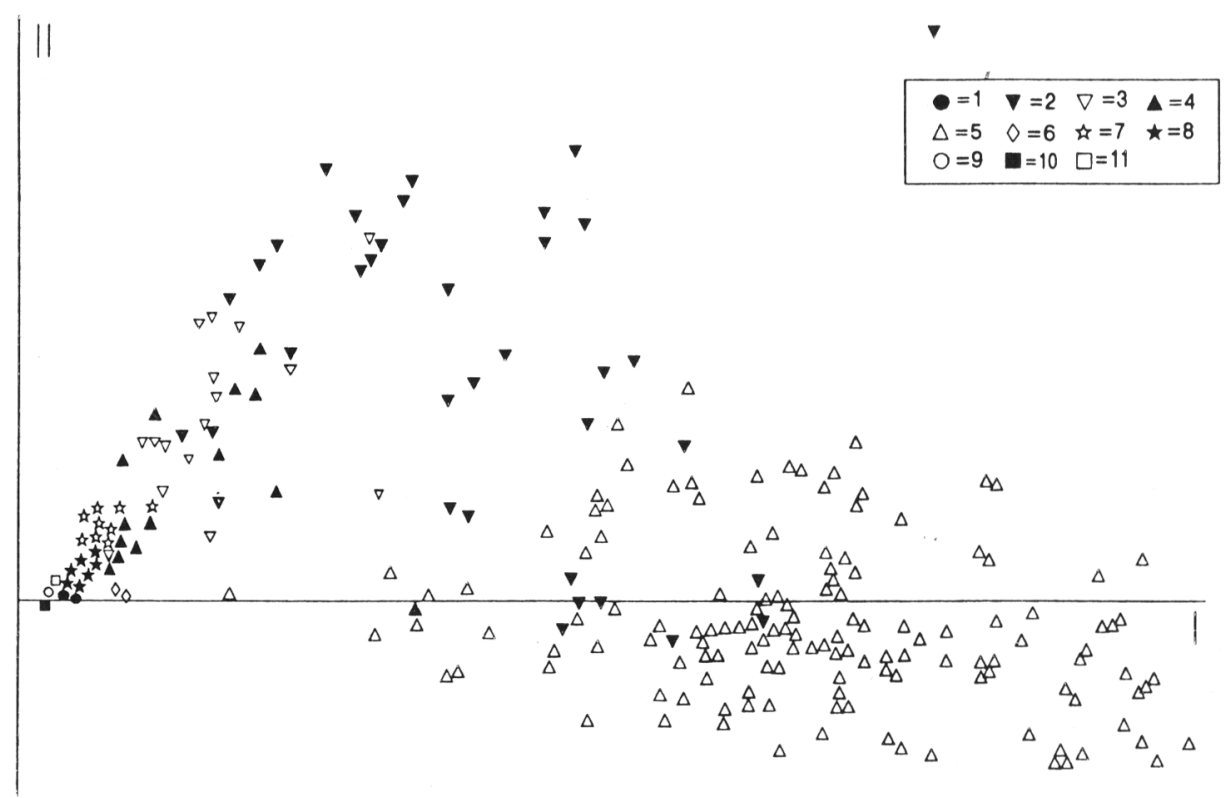

Figura 5. Diagrama de ordenación (PCA) de inventarios de pastos vivaces y anfibios (Juncetea bufonii). 1-Deschampsio strictae-Agrostietum curtisii, 2-Trifolio subterranei-Plantaginetum serrariae, 3-Gaudinio fragilis-Agrostietum castellanae, 4-Pulicario paludosae-Agrostietum pourretii, 5-Hedysaro coronariiPhalaridetum coerulescentis, 6-Centaureo exaratae-Armerietum gaditanae, 7-Loto subbifloriChaetopogonetum fasciculati, 8-Laurentio-Juncetum tingitani, 9-Heliotropio supini-Paspaletum paspaloidis, 10-Cypero micheliani-Heleochloetum alopecuroidis, 11-Glino lotoidis-Verbenetum supinae.Ordination $(P C A)$ diagram of relevés of perennial and amphibious (Juncetea bufonii) grassiands.

verano, con suelos muy arcillosos salobres. Esta naturaleza de los suelos hace que sea una asociación ligada al dominio climácico de los tarayares de Tamarix canariensis. La presencia en los inventarios de ciertas plantas como Eryngium uquifolium, Juncus maritimus, Panicum repens o Trifolium repens, permiten llevarla a la alianza Molinio-Holoschoenion. Desde el punto de vista corológico su distribución es aljíbica (subsector Sidonense), aunque en el norte de Africa probablemente existan asociaciones semejantes (Nègre, 1959; Thiault, 1957), al ser Festuca arundinacea subsp. atlantigena un taxon ibero-mauritánico (Jahandiez \& Maire, 1931).

En áreas con aluviones salinos margosos de vaguadas inundadas intermitentemente, reconocemos a la subasociación scirpetosum maritimi [typus: tabla 1, invent. 47].

2.4. Gaudinio fragilis-Agrostietum castellanae hypochaeridetosum platylepidis subass. nova

[Typus: Tabla 1, invent. 54]

La asociación Gaudinio fragilisAgrostietum castellanae ya fue descrita por Rivas-Martínez \& Belmonte (1985) para designar a los vallicares sobre suelos silíceos areno-limosos con un horizonte superficial pétreo. Su composición florística denota el cierto hidromorfismo que aparece en los claros de los alcornocales. La subasociación hypochaeridetosum platylepidis es termomediterránea y aljíbica. Sus plantas diferenciales territoriales son: Anthoxanthum ovatum subsp. ovatum, Armeria hirta, 


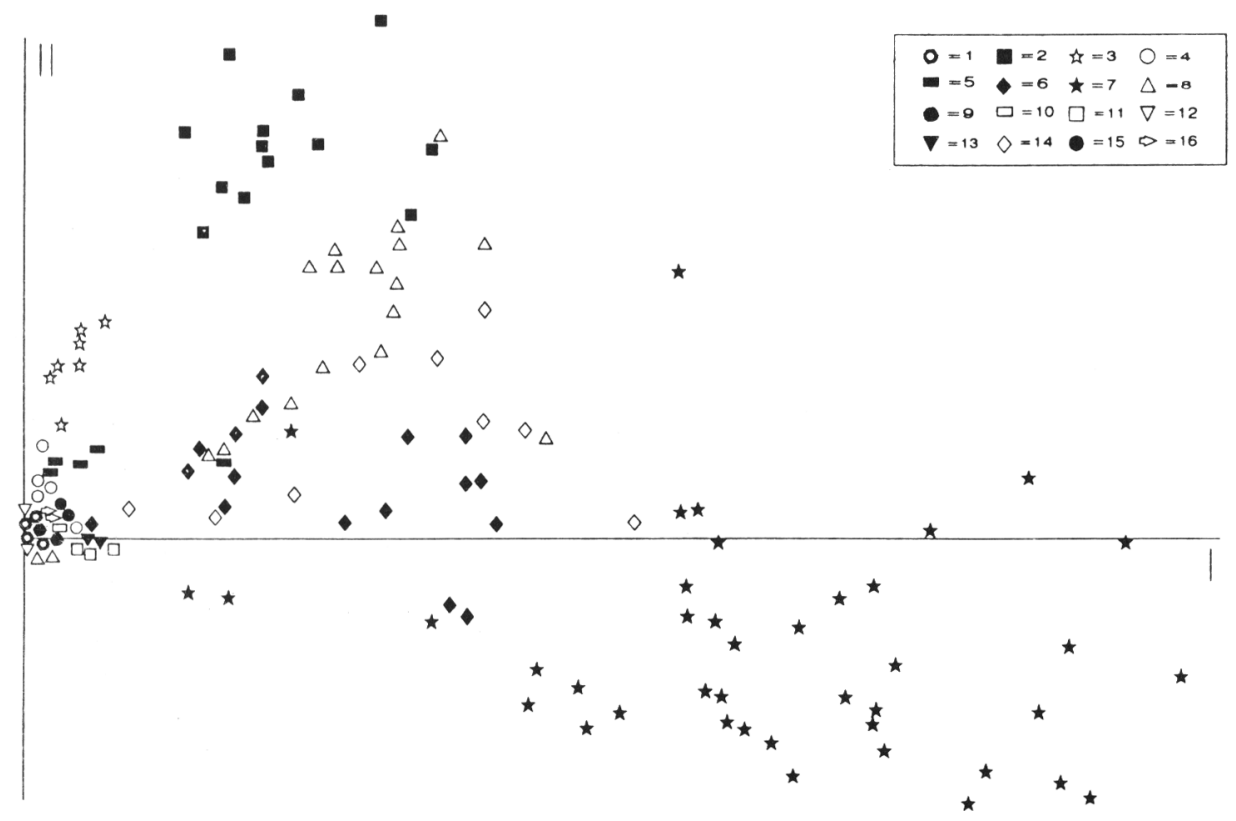

Figura 6. Diagrama de ordenación (PCA) de inventarios de comunidades nitrófilas. 1-Plantagini coronopiHordeetum marini, 2-Ridolfio segetum-Capnophylletum peregrini, 3-Chrozophoro tinctoriae-Teucrietum spinosi, 4-Chenopodietum muralis, 5-Arenario hispanicae-Chamaemeletum fuscati, 6-Anacyclo radiatiHordeetum leporini, 7-Trifolio pallidi-Vulpietum geniculatae, 8-Convolvulo meonanthi-Hedysaretum coronarii, 9-Crassulo tillaeae-Saginetum apetalae, 10-Gymnostyletum stoloniferae, 11-Linario viscosaeCarduetum meonanthi, 12-Geranio purpurei-Galietum minutuli, 13-Geranio rotundifolii-Theligonetum cynocrambis, 14-Urtico dubiae-Smyrnietum olusatri, 15-Kickxio lanigerae-Tanacetetum annui, 16-Com. Setaria verticillata-Echinochloa hispidula.Ordination (PCA) diagram of relevés of nitrofilous communities.

Hypochaeris radicata subsp. platylepis, Oenanthe globulosa, O. pimpinelloides, Ranunculus macrophyllus, Trifolium baeticum y T. pallidum. La presencia, a veces frecuente, de Plantago serraria demuestra el paso hacia las comunidades de Poetea bulbosae sobre arcillas y constante pisoteo del ganado vacuno.

\subsection{Ridolfio segeti-Capnophylletum peregrini Guinochet 1977}

[Capnophyllo peregrini-Ridolfietum segeti pro nom. inv.; Sin.: Bupleuro lancifoliiRidolfietum segeti Peinado, Martínez Parras \& Alcaraz 1989]

Asociación meseguera termomesomediterránea seco-subhúmedo-húmeda, presidida por Ridolfia segetum, que coloniza depresiones arcillosas tirsificadas, sobre las que se asientan cultivos cerealistas o con aprovechamiento con otras plantas (remolacha, girasol, garbanzos, etc.).

Entre los inventarios que hemos levantado no hemos encontrado diferencias significativas con la tabla de Guinochet (1977), ni con la de Peinado et al. (in Bartolomé et al., 1989) por lo que sinonimizamos Bupleuro-Ridolfietum segeti a Ridolfio-Capnophylletum peregrini.

Por otra parte, reconocemos a la alianza Ridolfion segeti, que reune a las asociaciones mesegueras de suelos vérticos (Nègre, 1956 y 1977; El Antri, 1983 y 1985) suribéricomauritánicos. Son taxones característicos: Bupleurum lancifolium, Capnophyllum peregrinum, Phalaris paradoxa, Ridolfia segetum y Silene stricta. Según esto, dentro 
in - oino oon -n

in o oino $\mathrm{OO}_{-}-\ldots$

ta ono oo- - .

$+\infty$ on 0 or $-1-0$

tr oino oo- hin

to ono oon -1

tin ono $00+-\infty$

t t o n o o On O O

$+m-00000-0$

$+n-0,00000$

th- ho

to-00 000 in

ma $-00000 \mathrm{no}$

$m x-00000 \mathrm{in}-$

mi - $00000 \mathrm{in}$

mo to o o o o mo

$m n-00000+n$

$m+-00000+m$

$m m-00000+0$

$m \mathrm{~m}-0000070$

$m-$ - 00000 in

mo - o o o e tr

na $-00000+7$

$n x-00000$ in -

nt $-00000+m$

ab-oO $000 \mathrm{mo}$

in -00 o o o in

$n+-000000-1$

$n m-00000$ n

$n h-00000+n$

$\mathrm{AH}-\mathrm{OO} 0 \mathrm{O}=\mathrm{O}$

no-oo o-in nm

- a o ino oino - 1

$-\infty$ oino o-o nin

-n - o o omo nn

-0 - 00 ono n-

- in - o o ono nn

at omo omo nt

$-m-000-m \mathrm{Nin}$

H h - $-0,0-0$ nin

- 0 - $00000 \mathrm{n}$

on to 0 in 0 -

$0 x-000 \times 0 \mathrm{~m}-$

or - o o p-x ma

0 too omrm-

On OOn-Oa OO

ot oor oar or

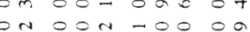

O- OOH-OO

$|1|$

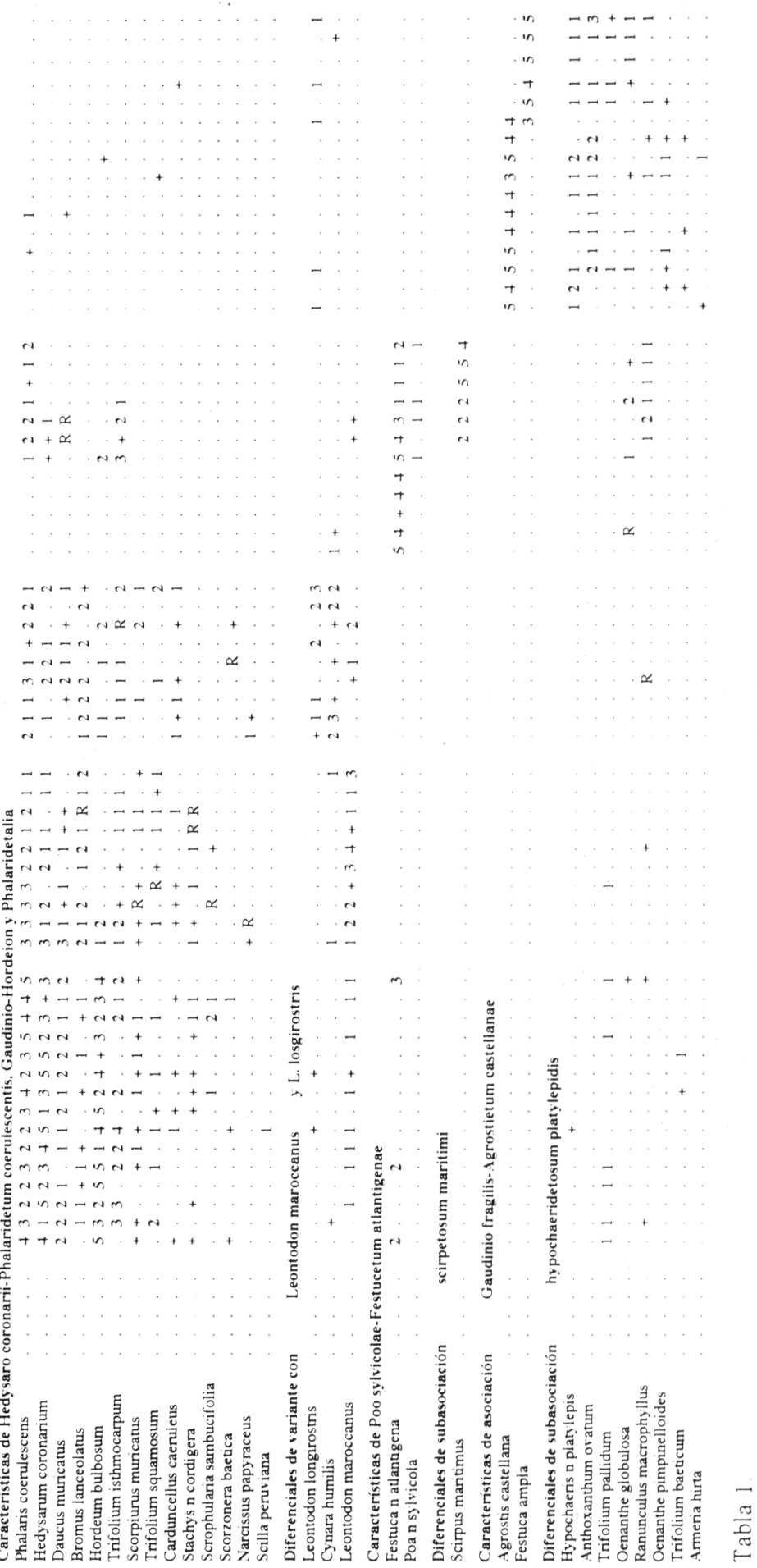




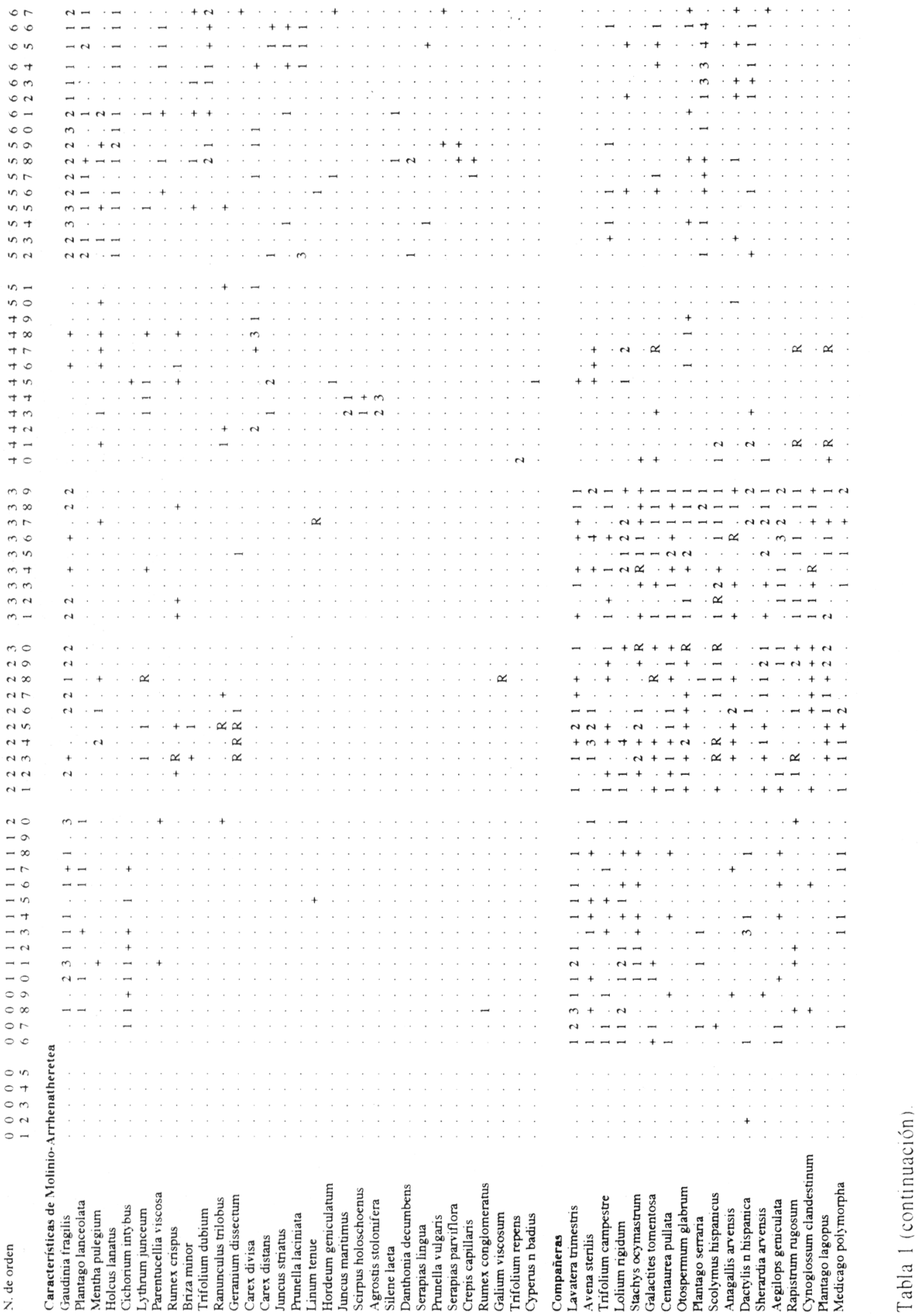




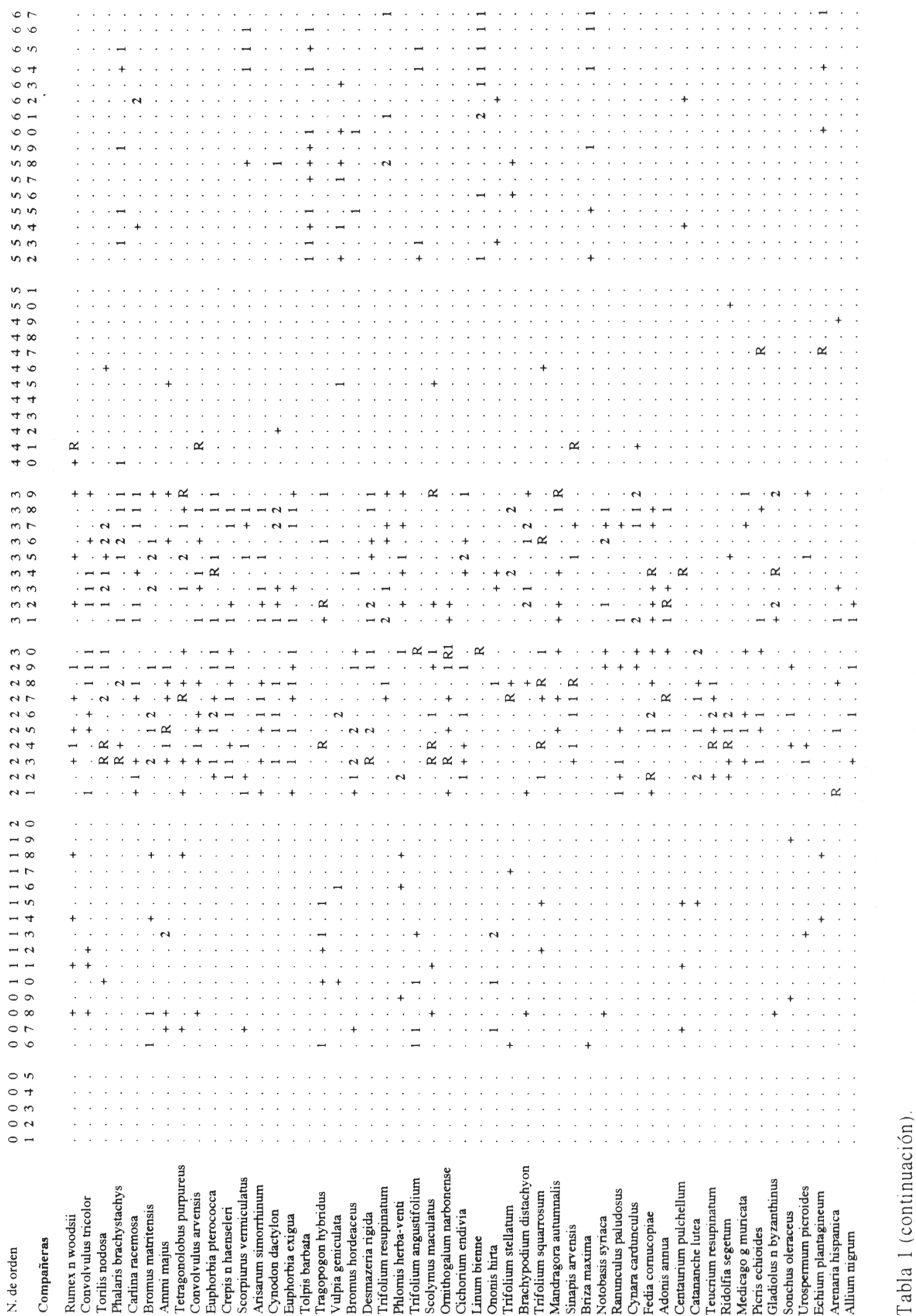




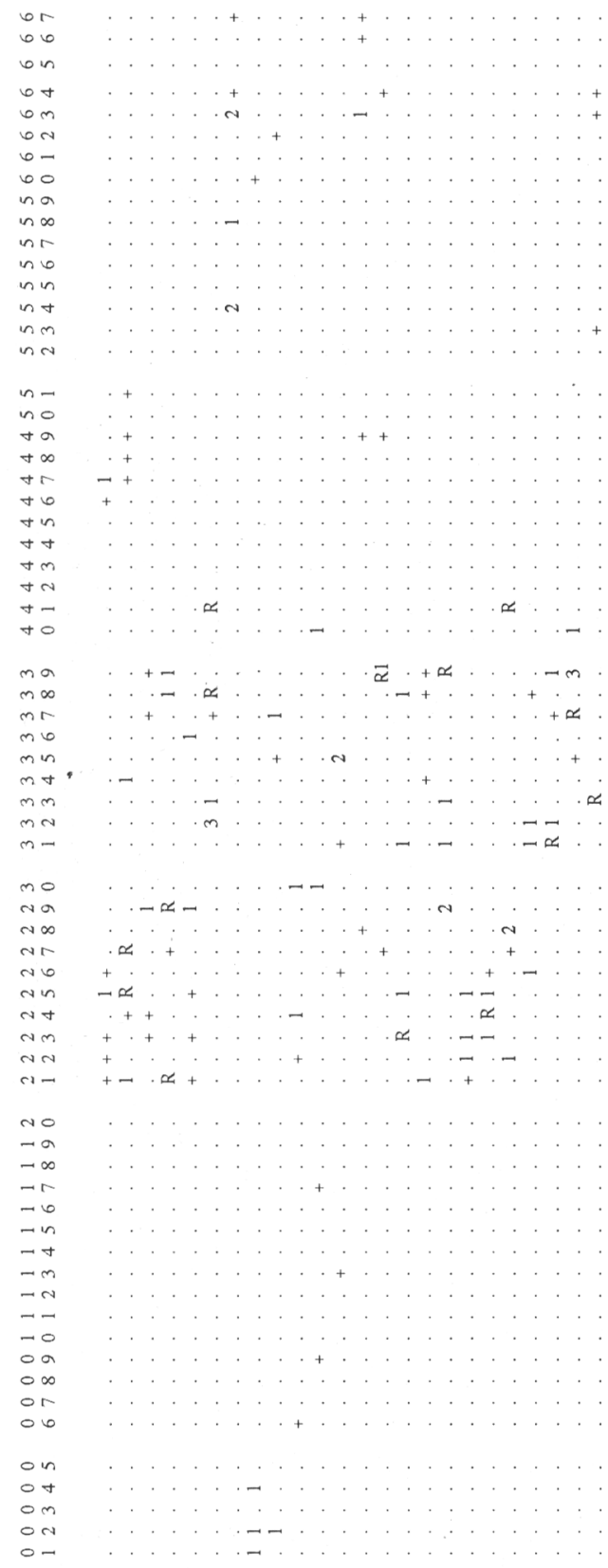

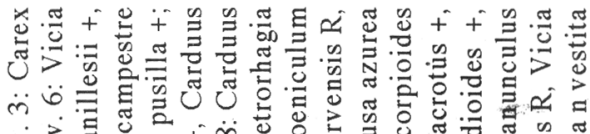

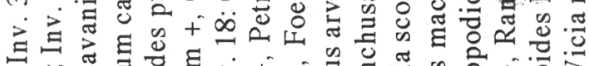

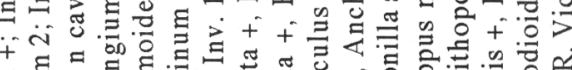

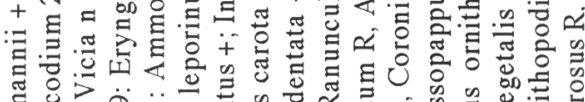

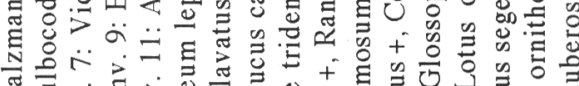

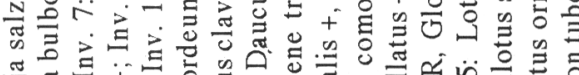

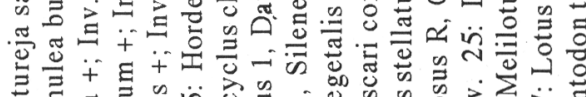

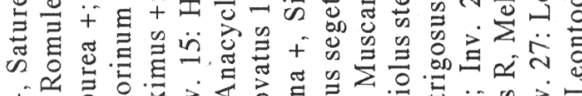

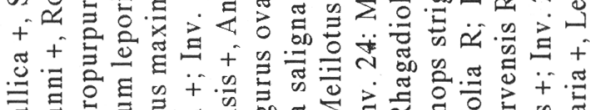

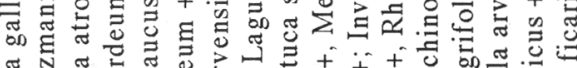

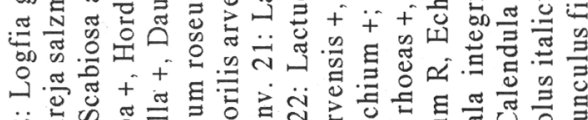

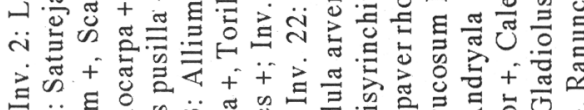

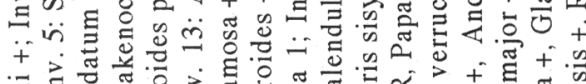

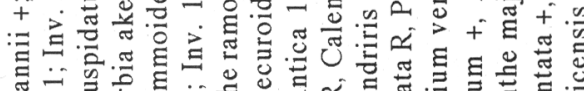

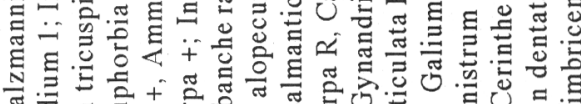

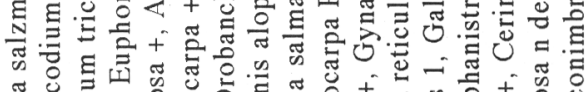

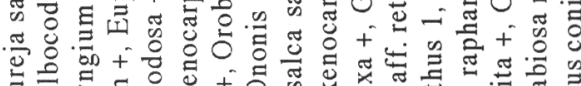

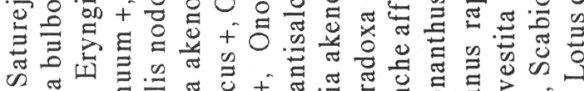

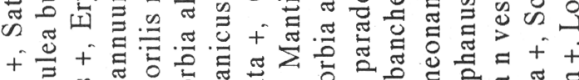

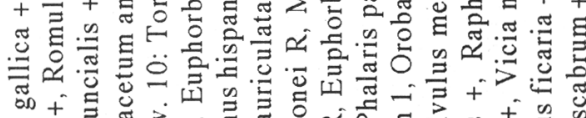

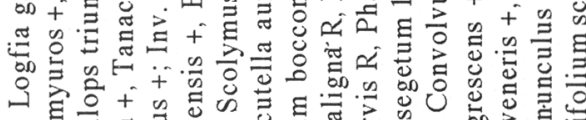

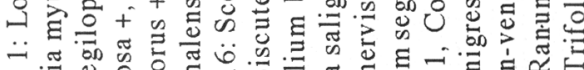

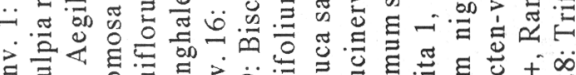

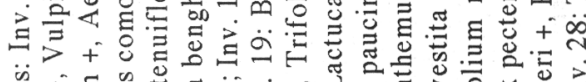

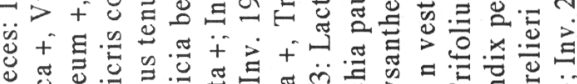

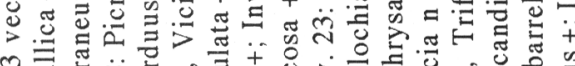
m.

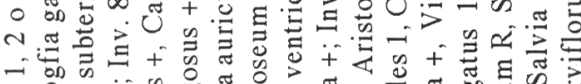

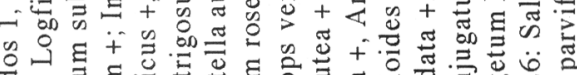

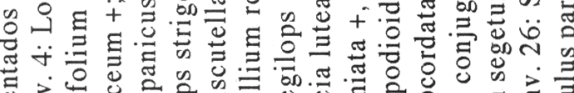

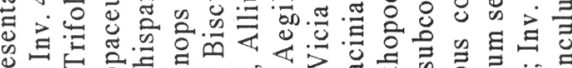

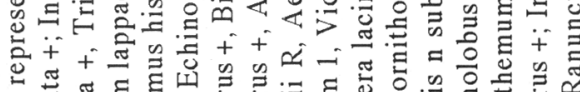

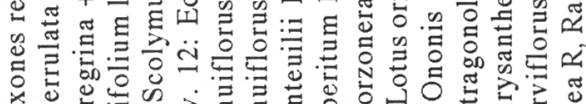

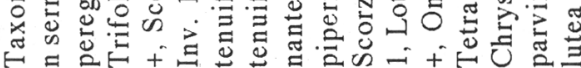




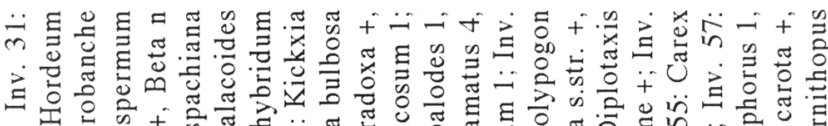

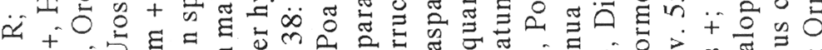

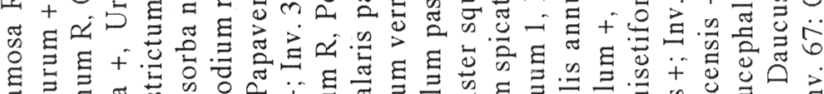

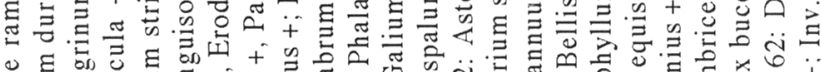

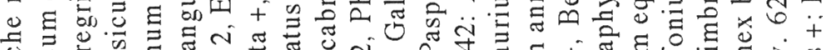

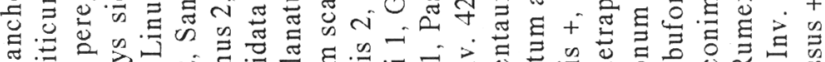

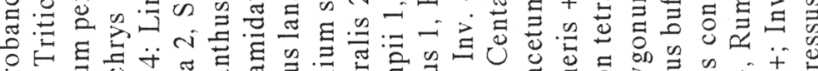

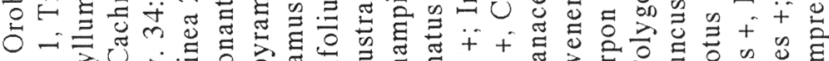
: :

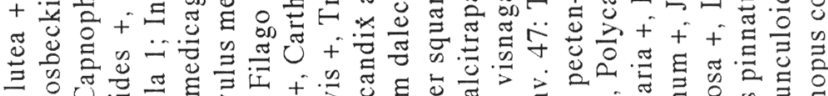

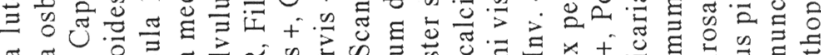
푼.

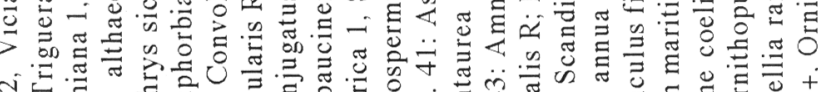
तE.

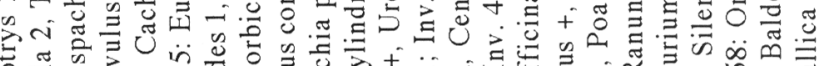

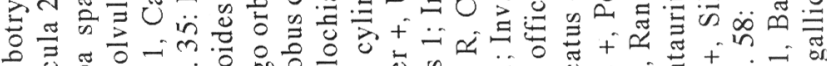

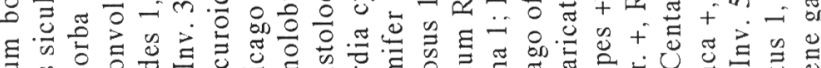

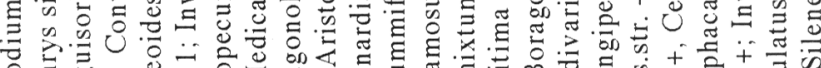

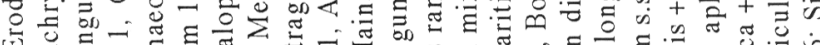

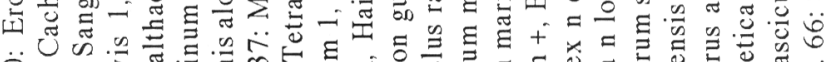

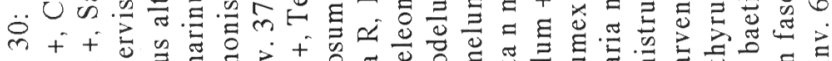

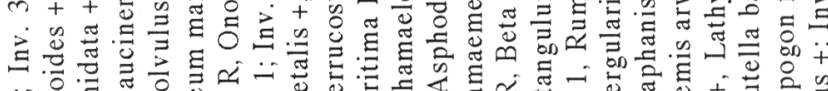

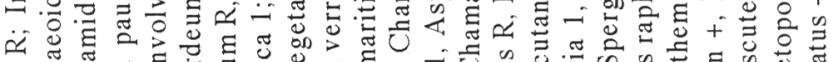

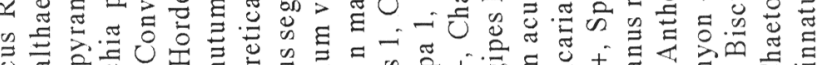

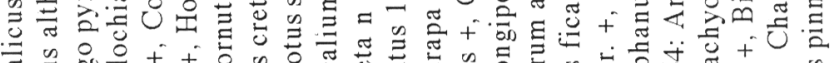

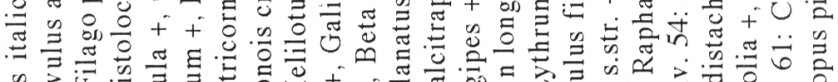

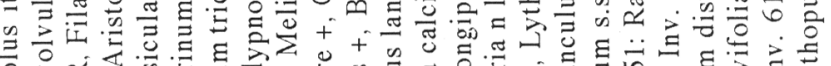

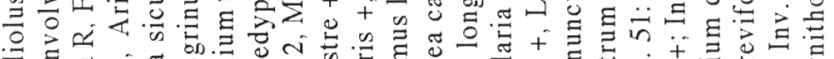

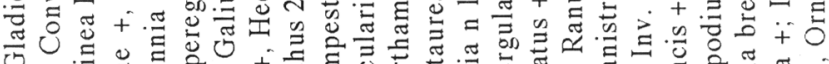

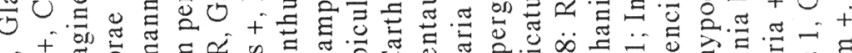

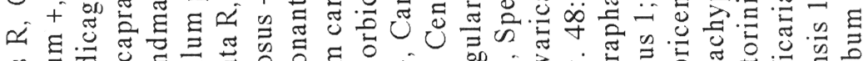

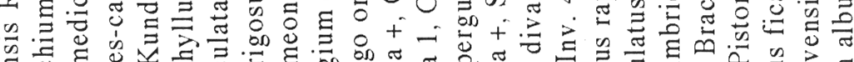

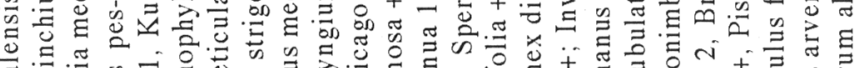

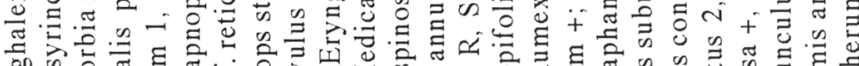

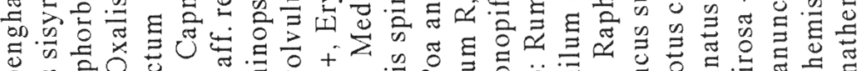
壱 志局

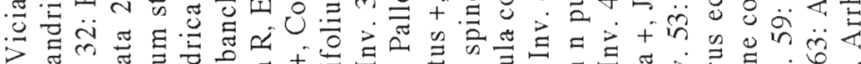

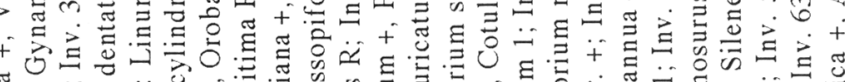

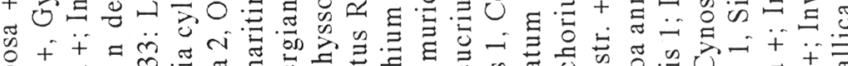

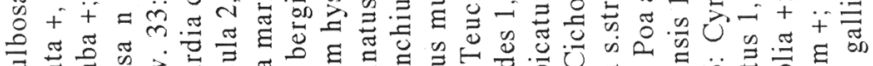

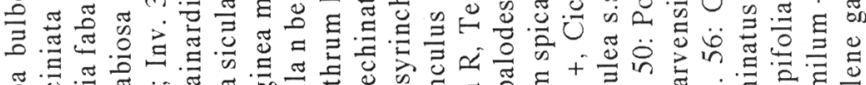

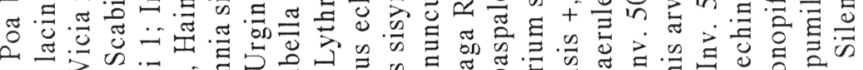

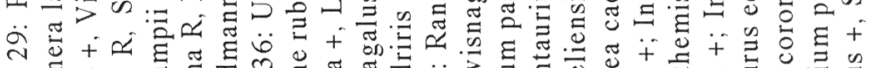

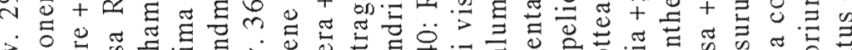

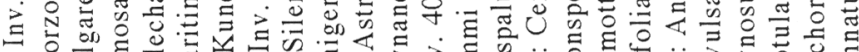

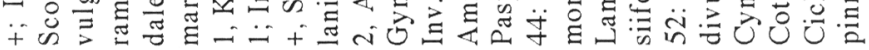

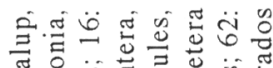

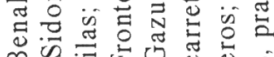
๓

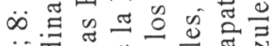

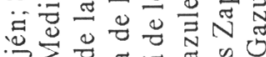

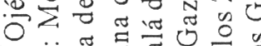

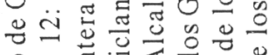

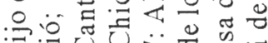

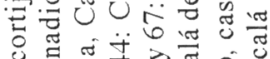

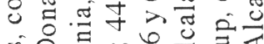
की

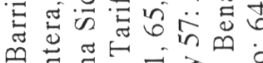

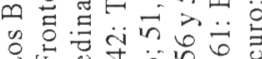

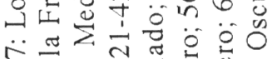

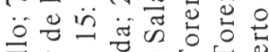

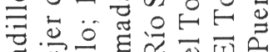

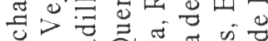

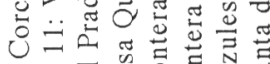

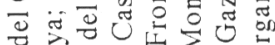
क

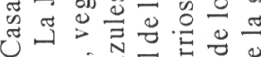
की क సٓ

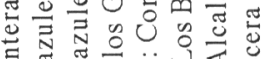

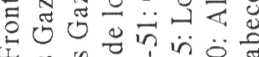
庄 ข

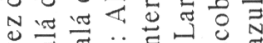

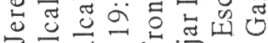

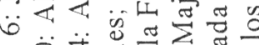

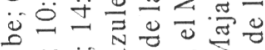

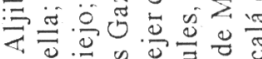

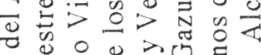

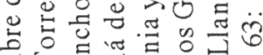
हึ。

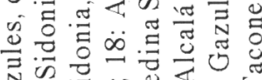
N刃 क क क

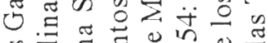

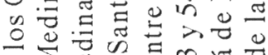

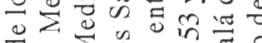

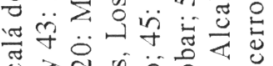

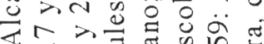

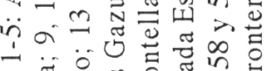

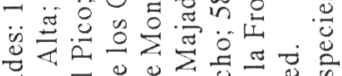

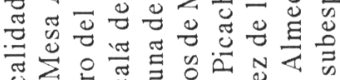

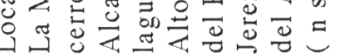




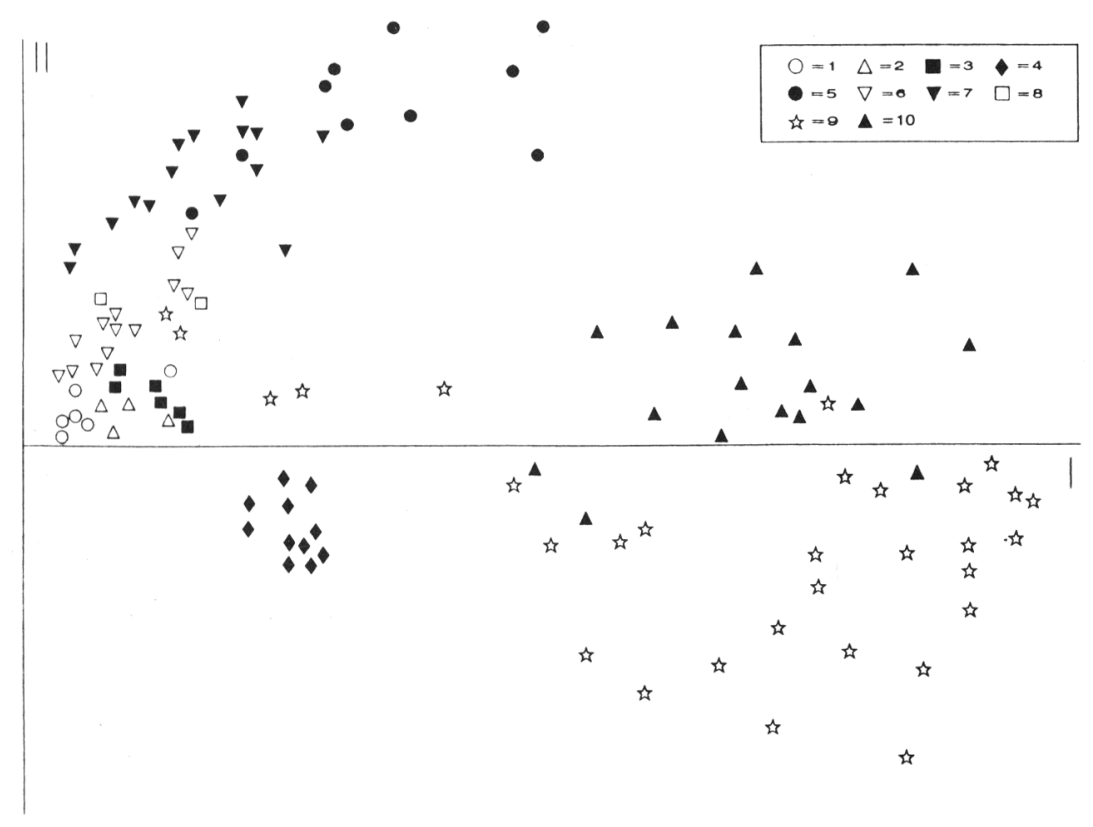

Figura 7. Diagrama de ordenación (PCA) de inventarios de juncales. 1-Com. Hydrocotyle vulgaris-Juncus subnodulosus, 2-Juncetum rugoso-effusi, 3-Trifolio resupinati-Caricetum chaetophyllae, 4-Glycerio declinatae-Eleocharitetum palustris, 5-Poo sylvicolae-Festucetum atlantigenae, 6-Junco emmanuelisEleocharitetum multicaulis, 7-Glycerio declinatae-Apietum nodiflori, 8-Galio palustris-Juncetum maritimi, 9-Holoschoeno-Juncetum acuti, 10-Astero squamati-Panicetum repentis. Ordination (PCA) diagram of relevés of rush wet places.

del orden Secaletalia cerealis podemos distinguir tres alianzas: Caucalidion platycarpae Tüxen (1937) 1950, de distribución atlántico-medioeuropea y supramediterránea septentrional, Secalion cerealis Br.-Br. 1931, meso- y supramediterránea meridional, y Ridolfion segeti (Nègre 1977) El Antri 1983, termo- y mesomediterránea inferior.

\section{CONCLUSIONES}

Como síntesis del trabajo, damos a conocer el esquema sintaxonómico comentado de los pastizales del área estudiada:

\section{CORYNEPHORETEA CANESCENTIS Br.-} BI. \& R. Tx. 1943

Jasonio sessiliflorae-Koelerietalia crassipedis Rivas-Martínez \& Cantó 1986

Festucion elegantis Rivas-Martínez \&
Sánchez Mata in Rivas-Martínez, Fernández González \& Sánchez Mata 1986 Deschampsio strictae-Agrostietum curtisii ass. nova [Graminales xerófilos mesomediterráneos. Aljíbica]

POETEA BULBOSAE Rivas Goday \& RivasMartínez in Rivas-Martínez 1978

Poetalia bulbosae Rivas Goday \& RivasMartínez ex Rivas Goday \& Ladero 1970

Poo-Trifolion subterranei Rivas Goday (1959) Rivas Goday \& Ladero 1970

Trifolio subterranei-Plantaginetum serrariae Martín Osorio \& Galán de Mera 1996 [Majadales termomesomediterráneos. Bética y aljíbica]

MOLINIO-ARRHENATHERETEA R. Tx. 1937

Phalaridetalia coerulescentis ordo novo Gaudinio fragilis-Hordeion bulbosi all. nova 
Hedysaro coronarii-Phálaridetum coerulescentis ass. nova [Praderas sobre vertisuelos. Bética occidental, aljíbica y tingitana]. var. de Leontodon longirostris y Cynara humilis. var. de Leontodon maroccanus.

Holoschoenetalia Br.-Bl. (1931) 1947

Molinio-Holoschoenion Br.-Bl. (1931) 1947

Comunidad Hydrocotyle vulgarisJuncus subnodulosus [Juncal alterado por aporte de materiales calcáreos] Holoschoeno-Juncetum acuti RivasMartínez \& Costa in Rivas-Martínez, Costa,; Castroviejo \& Valdés 1980 [Juncales con escasa humedad edáfica. Iberomárroquí-atlántica]

Galio palustri-Juncetum maritimi RivasMartínez \& Costa in Rivas-Martínez, Costa, Castroviejo \& Valdés 1980 [Juncales sobre suelos arcillosos temporalmente encharcados por aguas dulces. Iberomarroquí-atlántica] Poo sylvicolae-Festucetum atlantigenae ass. nova [Depresiones encharcadas con suelos vérticos salobres y pequeños valles con agua salobre. Aljíbica (sidonense)] y scirpetosum maritimi subass. nova [en aguas más profundas y suelos margoso-salinos]

Molinietalia coeruleae W. Koch 1926

Juncion acutiflori Br.-Bl. in Br.-Bl. \& R. Tx. 1952

Juncetum rugoso-effusi Rivas-Martínez \& Costa in Rivas-Martínez, Costa, Castroviejo \& Valdés 1980 [Juncales de carácter atlántico sobre suelos profundamente gleyzados. TingitanoOnubo-Algarviense]

Plantaginetalia majoris R. Tx. \& Preising in R. Tx. 1950

Trifolio fragiferi-Cynodontion dactylionis Br.-Bl. \& O. Bolós 1957

Trifolio resupinati-Caricetum chaetophyllae Rivas-Martínez \& Costa in Rivas-Martínez, Costa, Castroviejo \& Valdés 1980 [Gramadal denso sobre suelos de textura arenosa. Iberomarroquí-atlántica]

Paspalo paspalodis-Agrostion semiverticillati Br.-Bl. 1952

Astero squamati-Panicetum repentis Martínez Parras \& Peinado Lorca 1993 [Graminal vivaz termófilo con plantas de origen tropical. Bética occidental y aljíbica (sidonense)]

Heliotropio supini-Paspaletum paspalodis Martínez Parras, Peinado, Bartolomé \& Molero Mesa 1988 [Prados de las graveras de los ríos. Bética occidental y aljíbica (sidonense)]

Agrostietalia castellanae Rivas-Martínez in Rivas-Martínez, Costa, Castroviejo \& Valdés 1980

Agrostion castellanae Rivas Goday 1957 corr. Rivas Goday \& Rivas-Martínez 1963 Gaudinio fragilis-Agrostietum castellanae Rivas-Martínez \& Belmonte 1986 hypochaeridetosum platylepidis subass. nova [Vallicar sobre suelos areno-limosos. Aljíbica]

Centaureo exaratae-Armerietum gaditanae Allier \& Bresset 1977 corr. Rivas-Martínez, Costa, Castroviejo \& Valdés 1980 [Pastizal vivaz sobre suelos arenosos encharcados temporalmente. Tingitano-Onubo-Algarviense]

\section{POLYGONO ARENASTRI-POETEA ANNUAE Rivas-Martínez 1975}

Polygono arenastri-Poetalia annuae R. Tx. in Géhu, Richard \& R. Tx. 1972

Polycarpion tetraphylli Rivas-Martínez 1975

Crassulo tillaeae-Saginetum apetalae Rivas-Martínez 1975 [Terófitos nitrófilos de suelos arenosos pisoteados. Atlántica e Iberomarroquí-atlántica] Gymnostyletum stoloniferae RivasMartínez 1975 corr. Ladero et al. 1983 
[Terófitos nitrófilos urbanícolas. LusoExtremadurense y Tingitano-OnuboAlgarviense]

\section{RUDERALI-SECALIETEA CEREALIS Br.-} Bl. 1936

Secalienea cerealis Rivas-Martínez, Báscones, T.E. Díaz, Fernández González \& Loidi 1991

Secalietalia cerealis Br.-Bl. 1931

Ridolfion segeti (Nègre 1977) El Antri 1983

Riḋolfio segetum-Capnophylletum peregrini Guinochet 1977 [Comunidades mesegueras sobre suelos vérticos. Bética, aljíbica, mauritánica y Sicilia] Solano nigri-Polygonetalia convolvuli (Sissingh ex Westhoff, Dijk \& Passier 1946) O. Bolós 1962 em. Brullo \& Marceno 1979 Panico-Setarion Sissing 1946

Comunidad de Setaria verticillataEchinochloa hispidula [Herbazales de cultivos irrigados]

Diplotaxion erucoidis Br.-Bl. 1931

Chrozophoro tinctoriae-Teucrietum spinosi Galán de Mera 1996 [Asociación estival de barbechos con suelos vérticos. Bética occidental, aljíbica (sidonense)] Kickxio lanigerae-Tanacetetum annui Galán de Mera 1996 [Asociación autumnal sobre vertisuelos inundables. Bética occidental, aljíbica (sidonense)]

Chenopodienea muralis Rivas-Martínez, Báscones, T.E. Díaz, Fernández González \& Loidi 1991

Chenopodietalia muralis Br.-Bl. (1931) 1936

Chenopodion muralis Br.-Bl. $1931 \mathrm{em} . \mathrm{O}$.

Bolós 1967

Chenopodietum muralis Br.-B1. \& Maire 1924 [Herbazales estivo-autumnales urbanícolas. Mediterránea occidental]

Sisymbrietalia officinalis J. Tx. in Lohmeyer et al. 1962 em. Rivas-Martínez, Báscones, T.E. Díaz, Fernández González \& Loidi 1991 Hordeion leporini Br.-Bl. (1931) 1947
Anacyclo radiati-Hordeetum leporini $\mathrm{O}$. Bolós \& Rivas-Martínez in RivasMartínez 1978 [Comunidad nitrófila viaria. Bética, tingitano-onuboalgarviense, luso-extremadurense meridional y tingitana]. Subas. chrysanthemetosum coronarii RivasMartínez 1978 [Medios enriquecidos en bases]

Bromenalia rubenti-tectori Rivas-Martínez \& Izco 1977

Cerintho majoris-Fedion cornucopiae Rivas-Martínez \& Izco ex Peinado, Martínez Parras \& Bartolomé 1986

Convolvulo meonanthi-Hedysaretum coronarii Peinado, Martínez Parras \& Bartolomé 1986 [Comunidad terofítica pionera de campos abandonados. Bética occidental y aljíbica]. Subas. glossopappetosum macroti Galán de Mera 1995 [Medios enriquecidos en bases]

Arenario hispanicae-Chamaemeletum fuscati Gálan de Mera 1995 [Terófitos de barbechos en vertisuelos arenosos en superficie; Bética occidental y aljíbica] Echio-Galactition tomentosae O. Bolós \& Molinier 1969

Trifolio pallidi-Vulpietum geniculatae Galán de Mera 1995 [Asociación subnitrófila de suelos areno-limosos. Aljíbica]

Linario viscosae-Vulpion alopecuroris Rivas-Martínez \& Izco 1977 ex RivasMartínez, Costa, Castroviejo \& Valdés 1980

Linario viscosae-Carduetum meonanthi Rivas-Martínez, Costa, Castroviejo \& Valdés 1980 [Asociación psammófila de suelos removidos. Tingitano-OnuboAlgarvien'se]

Geranio purpurei-Cardaminetalia hirsutae Brullo in Brullo \& Marcenó 1985

Geranio pusilli-Anthriscion caucalidis Rivas-Martínez (1975) 1978 
Geranio purpurei-Galietum minutuli Rivas-Martínez \& Costa in RivasMartínez, Costa, Castroviejo \& Valdés 1980 [Asociación escionitrófila psamófila. Gaditano-onubense]

Geranio rotundifolii-Theligonetum cynocrambis Rivas-Martínez \& Malato Beliz in Rivas-Martínez 1977 [Asociación escionitrófila de suelos areno-limosos o arcillosos. Bética y Tingitano-onubo-algarviense]

Urtico-Scrophularietalia peregrinae Brullo 1985

Allion triquetri O. Bolós 1957

Urtico dubiae-Smyrnietum olusatri (A. \& O. Bolós 1950) O. Bolós \& Molinier 1958 [Asociación escionitrófila de talla elevada. Mediterránea occidental]

\section{TRIFOLIO-GERANIETEA SANGUINEI}

\section{Th. Müller 1962}

Origanietalia vulgaris Th. Müller 1962

Origanion virentis Rivas-Martínez \& O.

Bolós in Rivas-Martínez, Díaz, Prieto, Loidi \& Penas 1984

Calamintho baeticae-Galietum scabri Pérez Latorre, Nieto Caldera \& Cabezudo 1993 [Pastizales humícolas de lindero. Bética occidental y aljíbica]. lamietosum flexuosi Pérez Latorre, Nieto Caldera \& Cabezudo 1993 [Vegetación bajo quejigares]. Subas. digitaletosum bocquetii Pérez Latorre, Nieto Caldera \& Cabezudo [Vegetación bajo alcornocales]

JUNCETEA BUFONII (Br.-Bl. \& R. Tx. 1943) Foucault 1988

Scirpetalia setacei Foucault 1988

Cicendion (Rivas Goday \& Borja 1961) Br.-B1. 1967

Laurentio-Juncetum tingitani Rivas Goday \& Borja 1968 [Comunidad anfibia terofítica de aguas efímeras superficiales. Monchiquense, aljíbica y tingitana]

Loto subbiflori-Chaetopogonetum fasciculati Rivas-Martínez, Costa, Castroviejo \& Valdés 1980 [Asociación anfibia terofítica de suelos arenosos o limo-arenosos con aguas estancadas. Tingitano-onubo-algarviense]

Agrostion pourretii Rivas Goday (1955) 1957 nom. mut. Rivas-Martinez et al. 1986

Pulicario paludosae-Agrostietum pourretii Rivas Goday 1955 nom. mut. Rivas-Martinez et al. 1986 [Vallicares anuales de suelos areno-limosos con hidromorfía temporal. Iberomarroquíatlántica]. holcetosum lanati Rivas Goday 1957 [Subasociación de tránsito hacia los vallicares vivaces de Agrostis castellana]

Nanocyperetalia Klika 1935

Heleochloo-Cyperion (Br.-Bl. 1952) Pietsch 1961

Cypero micheliani-Heleochloetum alopecuroidis Rivas Goday \& Valdés in Rivas Goday 1970 [Asociación tardoestival nitrificada, con encharcamiento prolongado de orillas de embalses. Iberomarroquí-atlántica]

Verbenion supini Slavnic 1951

Glino lotoidis-Verbenetum supinae Rivas Goday 1964 [Asociación tardoestival nitrificada, con encharcamiento fugaz de orillas de embalses. Iberomarroquí-atlántica]

LITTORELLETEA Br.-BI. \& R. Tx. 1943

Littorelletalia W. Koch 1926

Hyperico helodis-Sparganion Br.-Bl. \& R. Tx. ex Oberdorfer 1957

Junco emmanuelis-Eleocharitetum multicaulis Rivas-Martínez \& Costa in Rivas-Martínez, Costa, Castroviejo \& Valdés 1980 [Vegetación anfibia vivaz de pequeños helófitos sobre sustratos hidroturbosos. Tingitano-onuboalgarviense] 


\section{PHRA G MITIO - M A GNOCAR I CETEA Klika in Klika \& Nowak 1941 \\ Magnocaricetalia Pignatti 1953 \\ Glycerio-Sparganion Br.-Bl. \& Sissingh in Boer 1942 \\ Glycerio declinatae-Eleocharitetum palustris Rivas-Martínez \& Costa in Rivas-Martínez, Costa, Castroviejo \& Valdés 1980 [Pastizal helofítico de aguas someras temporales. Iberomarroquí- atlántica] \\ Apion nodiflori Segal in Westhof \& Den Held 1969 \\ Glycerio declinatae-Apietum nodiflori Molina 1996 [Berreras nitrificadas de fuentes y bordes de arroyos. Iberomarroquí-atlántica]}

\section{FRANKENIETEA PULVERULENTAE Rivas-Martínez ex Castroviejo \& Porta 1976 em. Brullo 1988}

Frankenietalia pulverulentae Rivas-Martínez ex Castroviejo \& Porta 1976 em. Brullo 1988

Frankenion pulverulentae Rivas-Martínez ex Castroviejo \& Porta 1976 em. Brullo 1988

Plantagini coronopi-Hordeetum marini O. Bolós \& Molinier ex O. Bolós 1962 [Vegetación halonitrófila anual pastoreada y sometida a inundaciones temporales. Mediterránea occidental].

\section{BIBLIOGRAFÍA}

BARBERO, M., P. QUÉZEL \& S. RIVASMARTÍNEZ. - 1981-Contribution à l'étude des groupements forestiers et préforestiers du Maroc. Phytocoenol. 9(3): 311-412.

BARTOLOMÉ, C., M. PEINADO, J.M. MARTÍNEZ PARRAS, F. ALCARAZ, J. ÁLVAREZ y M. DE LA CRUZ. -1989-Esquema sintaxonómico de la vegetación nitrófila de Andalucía (España). Alcalá de Henares.

BRAUN-BLANQUET, J. -1979- Fitosociología. Bases para el estudio de las comunidades vegetales. Madrid.

BRAY, J.R. \& J.T. CURTIS. -1957- An ordination of the upland forest communities of southern Wisconsin. Ecol. Monogr. 27: 325-349.

CARBALLAS, T., F. MACÍAS, F. DÍAZ-FIERROS y J.A. ORTIZ-URRUTIA. -1981-Clave para la clasificación de los suelos. Utilizada en el mapa de suelos del Mundo de la FAO-UNESCO. Madrid.

CHAuve, P. -1968- Étude géologique du Nord de la Province de Cadix. Mem. IGME 60: 1-377.

DE LA CRUZ, G. -1991- ANACOM, sistema de análisis de comunidades. CINVESTAV-IPN, Unidad Mérida, México.

DIERSCHKE, H. -1995- Syntaxonomical survey of Molinio-Arrhenatheretea in Central Europe. Col. Phytosociol. 23: 387-399. Berlín.

EL ANTRI, M. -1983-Contribution à l'étude des groupements commensaux des cultures du Maroc. Aspects synsystématiques et agronomiques. Thése Univ. Paris-Sud. Orsay.

EL ANTRI, M. -1985-Approche synsystématique des groupements commensaux des cultures du Maroc. Col. Phytosociol. 12:283-311. Stuttgart.

FERNÁNDEZ ALÉS, R., M.J. LEIVA y J. LAFFARGA. -1991- Los pastizales del Campo de Gibraltar (Cádiz). Composición florística y calidad. Bol. R. Soc. Esp. Hist. Nat. (Sec. Biol.) 87 (1-4): 61-72.

GALÁN DE MERA, A., J.A. LUCAS, A. PROBANZA y J.A. VICENTE. -1995- Análisis multivariante de las comunidades terofíticas pioneras de la provincia de Cádiz. (España). Orsis 11: 141-153.

GALÁN DE MERA, A. -1995- Trifolio pallidiVulpietum geniculatae, nueva asociación del Echio-Galactition para el SW de la Península Ibérica. Doc. Phytosociol. 15 : 335-339.

GALÁN DE MERA, A. -1996- Datos sobre las comunidades de la alianza Diplotaxion erucoidis en el SW de la Península Ibérica. Investigaciones de la Biosfera 1: 11-14.

GALÁN DE MERA, A. \& J.A. VICENTE ORELLANA. -1996- Phytosociological study of the plant communities with Stauracanthus boivinii of the SW of the Iberian Peninsula and NW of Africa, using multivariate analysis. Bot. Helv. $106:$ 45-56.

GONZÁLEZ, J., C.F. BERMEJO, M. LADERO, S. RIVAS GODAY y A. HOYOS. -1973- Estudio 
fitoedafológico de los pastizales cespitosos de Poa bulbosa L. Anales Edaf. Agrobiol. 32(3,4): 185-231.

GONZÁLEZ DE TANAGO, A., J.A. RUIZ CORNEJO y F. ORTIZ. - 1980-Ensayos sobre comportamiento productivo de la zulla (Hedysarum coronarium L.). Pastos 10(2): 95-103.

GOUNOT, M. -1958- Contribution à l'étude des groupements végétaux messicoles et rudereaux de la Tunisie. Ann. Serv. Bot. Tunisie 31: 32-244.

GUINOCHET, M. -1977- Contribution à la synsystématique des pelouses therophytiques du nord de la Tunisie et de l'Algérie. Col. Phytosociol. 6: 1-21.

HOLZNER, W., M.J.A. WERGER \& G.A. ELLENBROEK. -1978- Automatic classification of phytosociological data on the basis of species groups. Vegetatio 38: 157-164.

JAHANDIEZ, E. \& R. MAIRE. -1931- Catalogue des plantes de Maroc, I. Alger.

KOCH, W. -1926- Die Vegetationseinheiten der Linthebene unter Berücksichtigung der Verhältnisse in der Nordostschweiz. St. Gallen.

MOLINA, J.A. -1996- Sobre la vegetación de los humedales de la Península Ibérica (1. PhragmitiMagnocaricetea). Lazaroa $16: 27-88$.

MONTSERRAT, P. -1981-El césped y su dinamismo. Stu. Oecologica 1(1): 13-24.

MOORE, J.J. \& A.M. SULLIVAN. -1978- A phytosociological survey of the Irish MolinioArrhenatheretea using computer techniques. Vegetatio 38: 89-93.

MUDARRA GÓMEZ, J.L., BARAHONA FERNÁNDEZ, C. BAÑOS MORENO, A. IRIARTE MAYO y F. SANTOS FRANCES. 1989- Mapa de suelos de Andalucía. Madrid.

NÈGRE, R. -1956-Recherches phytosociologiques sur le Sedd-el-Messjoun. Trav. Inst. Sci. Chérifien, sér. Bot. 10:1-190.

NÈGRE, R. -1959- Recherches phytosociologiques sur l'étage de végétation méditérranéen aride (sous-étage chaud) au Maroc occidental. Trav. Inst. Sci. Chérifien, sér. Bot. 13: 1-385. Rabat.

NÈGRE, R. -1977- Donnees phytosociologiques sur les associations therophytiques du Maroc aride. Col. Phytosociol. 6: 23-32.

NEZADAL, W., U. DEIL \& W. WELB. -1994Karte der aktuellen Vegetation des Campo de Gibraltar (Provinz Cádiz, Spanien). Hoppea, Denkschr. Regensb. Bot. Ges. 55: 717-756.
OBERDORFER, E. -1990-Pflanzensoziologische Excursionsflora. Stuttgart.

ORLÓCI, L. -1978-Multivariate analysis in vegetation research. The Hague.

PÉREZ LATORRE, A.V., A. GALÁN DE MERA, U. DEIL y B. CABEZUDO. - 1996- Fitogeografía y vegetación del sector Aljíbico (Cádiz-Málaga, España). Acta Bot. Malacitana 21: 241-267.

PIELOU, E.C. -1969- An introduction to mathematical ecology. New York.

RIVAS GODAY, S. - 1964-Vegetación y flórula de la cuenca extremeña del Guadiana. Madrid.

RIVAS GODAY, S. y S. RIVAS-MARTÍNEZ. 1963-Estudio y clasificación de los pastizales españoles. Madrid.

RIVAS-MARTÍNEZ, S. -1979- Brezales y jarales de Europa occidental (Revisión fitosociológica de las clases Calluno-Ulicetea y CistoLavanduletea). Lazaroa 1: 5-128.

RIVAS-MARTÍNEZ, S. -1987-Memoria y mapa de las series de vegetación de España. Madrid.

RIVAS-MARTÍNEZ, S., A. ASENSI, J. MOLERO MESA y F. VALLE. -1991a- Endemismos vasculares de Andalucía. Rivasgodaya 6: 5-76.

RIVAS-MARTÍNEZ, S., J.C. BÁSCONES, T.E. DÍAZ, F. FERNÁNDEZ GONZÁLEZ y J. LOIDI. -199lb- Vegetación del Pirineo occidental y Navarra. Itinera Geobot. 5: 5-456.

RIVAS-MARTÍNEZ, S. y D. BELMONTE. -1986Sobre el orden Agrostietalia castellanae. Lazaroa 8: 417-419.

RIVAS-MARTÍNEZ, S., M. COSTA, S. CASTROVIEJO y E. VALDÉS. -1980Vẻgetación de Doñana (Huelva, España). Lazaroa 2: 5-189.

RIVAS-MARTÍNEZ, S., T.E. DÍAZ, J.A.F PRIETO, J. LOIDI y A. PENAS. -1984- La vegetación de la alta montaña cantábrica. Los Picos de Europa. León.

RIVAS-MARTÍNEZ, S., F. FERNÁNDEZ GONZÁLEZ y D. SÁNCHEZ MATA. -1986Datos sobre la vegetación del Sistema Central y Sierra Nevada. Opusc. Bot. Pharm. Complutensis 2: 3-136.

RIVAS-MARTÍNEZ, S., M. LOUSA, T.E. DÍAZ, F. FERNÁNDEZ GONZÁLEZ y J.C. COSTA. 1990- La vegetación del sur de Portugal (Sado, Alentejo y Algarve). Itinera Geobot. 3: 5-126.

SANTOS, M.T., M. LADERO y A. AMOR. -1989Vegetación de las intercalaciones básicas de la 
provincia de Cáceres (Extremadura, España). Studia Bot. 7: 9-147.

THIAULT, M. -1957-Les pelouses de la Tunisie du Nord et leurs aptitudes pastorales. Ann. Serv. Bot. Agron. Tunisie 30: 165-170.

THIAULT, M. -1961- Valeur pastorale des plantes fourragères spontanèes. Fourrages 6: 63-80.

TÜXEN, R. \& E. OBERDORFER. -1958Eurosibirische Phanerogamengesellschaften Spaniens. Veröff. Geobot. Inst. Rübel Zürich 32: 1-328.

VAN DER MAAREL, E. -1979- Transformation of cover-abundance values in phytosociology and its effects on community similarity. Vegetatio 39: 97-114.

Aceptado para su publicación en Junio de 1997

Dirección de los autores. A. GALÁN DE MERA y J.A. VICENTE ORELLANA: Departamento de Biología. Laboratorio de Botánica. Universidad San Pablo-CEU. Ap. 67. E-28660 Boadilla del Monte (Madrid). H. HAUG: Lehrstuhl Biogeographie. Universität Bayreuth. D-95440 Bayreuth. U. DEIL : Institut für Biologie II/Geobotanik. Universität Freiburg. Schänzlestraße 1. D-79104 Freiburg i.Br. 